ANALYSIS \& PDE Volume $5 \quad$ No. $2 \quad 2012$

ZAHER HANI

A BILINEAR OSCILLATORY INTEGRAL ESTIMATE AND BILINEAR REFINEMENTS TO STRICHARTZ ESTIMATES ON CLOSED MANIFOLDS 


\title{
A BILINEAR OSCILLATORY INTEGRAL ESTIMATE AND BILINEAR REFINEMENTS TO STRICHARTZ ESTIMATES ON CLOSED MANIFOLDS
}

\author{
ZAHER HANI
}

\begin{abstract}
We prove a bilinear $L^{2}\left(\mathbb{R}^{d}\right) \times L^{2}\left(\mathbb{R}^{d}\right) \rightarrow L^{2}\left(\mathbb{R}^{d+1}\right)$ estimate for a pair of oscillatory integral operators with different asymptotic parameters and phase functions satisfying a transversality condition. This is then used to prove a bilinear refinement to Strichartz estimates on closed manifolds, similar to that derived by Bourgain on $\mathbb{R}^{d}$, but at a relevant semiclassical scale. These estimates will be employed elsewhere to prove global well-posedness below $H^{1}$ for the cubic nonlinear Schrödinger equation on closed surfaces.
\end{abstract}

\section{Introduction}

We consider oscillatory integrals defined by

$$
T_{\lambda} f(t, x)=\int_{\mathbb{R}^{d}} e^{i \lambda \phi(t, x, \xi)} a(t, x, \xi) f(\xi) d \xi,
$$

where $t \in \mathbb{R}, x, \xi \in \mathbb{R}^{d}, a \in C_{0}^{\infty}\left(\mathbb{R} \times \mathbb{R}^{d} \times \mathbb{R}^{d}\right)$. The phase function $\phi$ is a real-valued smooth function on the support of $a$. We shall assume that it satisfies a usual nondegeneracy condition, namely that the $(d+1) \times d$ matrix

$$
\frac{\partial^{2} \phi}{\partial \xi \partial(x, t)}\left(t_{0}, x_{0}, \xi_{0}\right) \text { has maximal rank } d \text { for every }\left(t_{0}, x_{0}, \xi_{0}\right) \in \operatorname{supp} a .
$$

This implies that for each fixed $\left(t_{0}, x_{0}\right) \in \mathbb{R}^{d+1}$, the map given by

$$
\xi \mapsto \nabla_{(t, x)} \phi\left(t_{0}, x_{0}, \xi\right)
$$

defines a smooth immersion from $\mathbb{R}^{d}$ into $\mathbb{R}^{d+1}$. The image of this map is a hypersurface which we denote by $S_{\phi}\left(t_{0}, x_{0}\right)$, or just $S_{\phi}$ when no confusion arises. Our objective is to prove bilinear estimates for such operators and use them to get bilinear refinements to Strichartz estimates on compact manifolds without boundary.

Operators as in (1-1) can be thought of as variable coefficient generalizations of usual dual restriction (extension) operators where $\phi(t, x, \xi)=x . \xi+t \psi(\xi)$ and (1-1) becomes the dual of the operator given by restricting the Fourier transform to the hypersurface $S_{\phi}=\left\{(\tau, \xi) \in \mathbb{R}^{d+1}: \tau=\psi(\xi)\right\}$. As in the case of restriction operators, one is interested in obtaining asymptotic decay estimates for $\left\|T_{\lambda}\right\|_{L^{p}\left(\mathbb{R}^{d}\right) \rightarrow L^{q}\left(\mathbb{R}^{d+1}\right)}$ in terms of $\lambda$. It is well known that in order to obtain nontrivial decay estimates (the optimal one being

MSC2000: primary 35B45, 42B20, 58J40; secondary 35A17, 35S30.

Keywords: bilinear oscillatory integrals, bilinear Strichartz estimates, transversality, semiclassical time scale, nonlinear

Schrödinger equation on compact manifolds. 
$\lambda^{-(d+1) / q}$ ), one has to impose some curvature condition on the hypersurfaces $S_{\phi}$, namely that the Gaussian curvature does not vanish anywhere. The pairs of exponents $(p, q)$ for which this decay is possible were specified by Hörmander [1973] when $d=1$ and posed as a question for higher dimensions. Since then, there has been a tremendous amount of research in proving such bounds. (See [Stein 1993] and references therein for an introduction and [Tao 2004] for a more current survey).

We will be interested in bilinear versions of such estimates. In this case, one considers the product $T_{\lambda} f \tilde{T}_{\mu} g$, where $\tilde{T}_{\mu} g$ is an operator similar to (1-1):

$$
\tilde{T}_{\mu} g(t, x)=\int_{\mathbb{R}^{d}} e^{i \mu \psi(t, x, \xi)} b(t, x, \xi) g(\xi) d \xi,
$$

where $b \in C_{0}^{\infty}\left(\mathbb{R} \times \mathbb{R}^{d} \times \mathbb{R}^{d}\right)$ and $\psi$ is smooth on the support of $b$ and satisfies the same nondegeneracy assumption (1-2). The initial motivation behind such estimates was proving and refining the linear estimates in the case when the exponent $q$ is an even number. However, such an improvement is only possible when the surfaces $S_{\phi}$ and $S_{\psi}$ satisfy a certain transversality assumption. This transversality turns out to be more important than any curvature assumption in certain instances. To be precise, the type of estimates one is often interested in are of the form

$$
\left\|T_{\lambda} f \tilde{T}_{\mu} g\right\|_{L^{q}\left(\mathbb{R} \times \mathbb{R}^{d}\right)} \lesssim \Lambda(\lambda, \mu)\|f\|_{L^{2}\left(\mathbb{R}^{d}\right)}\|g\|_{L^{2}\left(\mathbb{R}^{d}\right)} .
$$

(For us, the case when $q=2$ and $\lambda \neq \mu$ will be of particular interest.) Great progress has been achieved in proving estimates like (1-4) especially in the case $\lambda=\mu$ and when the surfaces $S_{\phi}$ and $S_{\psi}$ satisfy some nonvanishing curvature assumption. In the constant coefficient (restriction) case, Wolff was able to prove (1-4) in the cone restriction case for all $q>1+2 /(d+1)$ with $\Lambda(\lambda, \lambda) \lesssim \lambda^{-(d+1) / q}$ [Wolff 2001]. This estimate was later extended to the endpoint in [Tao 2001]. The same estimate was then proven for transverse subsets of the paraboloid [Tao 2003]. In the variable coefficient case, Lee proved a similar estimate when $\lambda=\mu, q \geqslant 1+2 /(d+1)$, and $\Lambda(\lambda, \lambda) \lesssim \lambda^{-(d+1) / q+\epsilon}$ under certain curvature assumptions on the surfaces $S_{\phi}\left(t_{0}, x_{0}\right)$ and $S_{\psi}\left(t_{0}, x_{0}\right)$ [Lee 2006].

In this paper, we prove an $L^{2}$ estimate when $\lambda \neq \mu$ and the only assumption we impose on the hypersurfaces $S_{\phi}$ and $S_{\psi}$ is transversality. In particular, no curvature assumptions are taken.

Theorem 1.1. Suppose that $T_{\lambda}$ and $\tilde{T}_{\mu}$ are two oscillatory integral operators of the form given in (1-1) with $\mu \leqslant \lambda$ and assume that the canonical hypersurfaces associated with the phase functions $\phi$ and $\psi$ satisfy the standard transversality condition (1-6), then

$$
\left\|T_{\lambda} f \tilde{T}_{\mu} g\right\|_{L^{2}\left(\mathbb{R} \times \mathbb{R}^{d}\right)} \lesssim \frac{1}{\lambda^{d / 2} \mu^{1 / 2}}\|f\|_{L^{2}\left(\mathbb{R}^{d}\right)}\|g\|_{L^{2}\left(\mathbb{R}^{d}\right)} .
$$

The implicit constants are allowed to depend on $\delta, d$, and uniform bounds on a fixed number of derivatives of $\phi, \psi, a$, and $b$.

A couple of remarks are in order. First, we mention that (1-5) is sharp (see the remark at the end of Section 2). Second, we note that without curvature assumptions on the surfaces, the linear estimate is easily seen to fail (consider the restriction to hyperplanes). However, the $L^{2}$ bilinear estimate is true as 
long as the surfaces are transverse. ${ }^{1}$ Even when the linear estimate is true (which requires as mentioned a nonvanishing curvature assumption on the surfaces), (1-5) is an improvement on applying Hölder and the linear estimates available especially in the case when $\mu \ll \lambda$ (for example, when $d=2$ linear estimates give the bound $(\lambda \mu)^{-3 / 4}$ ). This improvement is often of great importance in applications (see [Bourgain 1999; 1998; Hani 2012]).

We now specify the transversality condition needed. The canonical hypersurfaces $S_{\phi}\left(t_{0}, x_{0}\right)$ and $S_{\psi}\left(t_{0}, x_{0}\right)$, given by the maps $\xi \mapsto \nabla_{(t, x)} \phi\left(t_{0}, x_{0}, \xi\right)$ and $\xi \mapsto \nabla_{(t, x)} \psi\left(t_{0}, x_{0}, \xi\right)$ respectively, live in the cotangent space $T_{\left(t_{0}, x_{0}\right)}^{*} \mathbb{R}^{d+1}$ to $\mathbb{R}^{d+1}$ at $\left(t_{0}, x_{0}\right)$. The nondegeneracy condition defined in (1-2) for $\phi$ (and defined similarly for $\psi)$, implies that for every $\xi_{0} \in \operatorname{supp}_{\xi} a\left(t_{0}, x_{0}, \cdot\right)$, there exists a locally defined unit normal vector field $v_{1}\left(t_{0}, x_{0}, \xi_{0}\right)=v_{1}\left(\xi_{0}\right)$ to this surface at the point $\nabla_{(t, x)} \phi\left(t_{0}, x_{0}, \xi_{0}\right) \in T_{\left(t_{0}, x_{0}\right)}^{*} \mathbb{R}^{d+1}$. In other words, the map

$$
\xi \mapsto\left\langle v_{1}\left(\xi_{0}\right), \nabla_{(t, x)} \phi\left(t_{0}, x_{0}, \xi\right)\right\rangle
$$

has a critical point at $\xi=\xi_{0}$ (in linear algebra terms, $v\left(\xi_{0}\right)$ is the unit vector spanning the one dimensional orthogonal complement of the image of the matrix appearing in (1-2)). Similarly, we define the associated unit normal vector $\nu_{2}\left(\xi_{0}\right)$ to $S_{\psi}\left(t_{0}, x_{0}\right)$ at the point $\nabla_{(t, x)} \psi\left(t_{0}, x_{0}, \xi_{0}\right)$ satisfying

$$
\xi \mapsto\left\langle v_{2}\left(\xi_{0}\right), \nabla_{(t, x)} \psi\left(t_{0}, x_{0}, \xi\right)\right\rangle
$$

has a critical point at $\xi=\xi_{0}$.

The transversality condition we impose on the phase functions $\phi$ and $\psi$ is that the two surfaces $S_{\phi}\left(t_{0}, x_{0}\right)$ with $S_{\psi}\left(t_{0}, x_{0}\right)$ are uniformly transverse for every $\left(t_{0}, x_{0}\right)$ : by which we mean that there exists a $\delta>0$ such that for each $\left(t_{0}, x_{0}, \xi_{1}\right) \in \operatorname{supp} a,\left(t_{0}, x_{0}, \xi_{2}\right) \in \operatorname{supp} b$, we have

$$
\left|\left\langle v_{1}\left(\xi_{1}\right), \nu_{2}\left(\xi_{2}\right)\right\rangle\right| \leqslant 1-\delta
$$

This transversality condition is standard in all bilinear oscillatory integral estimates. We remark that there is a slight difference between this definition of transversality and that used in most differential topology textbooks in which the definition of transversality includes manifolds that do not intersect. Here we say that two hypersurfaces are transverse if the intersection of all their translates is transverse in the sense of differential topology.

Remark. The phase functions $\phi$ and $\psi$ can depend on $\lambda$ and $\mu$ as long as the quantitative estimates needed in the proof (namely (1-6) and the derivative bounds mentioned in Equation (1-5)) are satisfied uniformly in $\lambda$ and $\mu$ on the support of $a$ and $b$.

The proof of Theorem 1.1 is based on a $T T^{*}$ argument and delicate analysis of a cumulative phase function.

Bilinear Strichartz estimates. Our main application of the bilinear estimate in Theorem 1.1 is to derive short-range or semiclassical bilinear Strichartz estimates for the Schrodinger equation on closed (compact

\footnotetext{
${ }^{1}$ This is well known in the constant coefficient case; see [Tao 2004].
} 
without boundary) $d$-manifolds $M^{d}$. We will also be able to prove mixed bilinear estimates of Schrödingerwave type as well (see Section 4). Bilinear estimates are of great importance in PDE as they offer refinements to linear Strichartz estimates. The latter are given on $\mathbb{R}^{d}$ with its Euclidean Laplacian by

$$
\left\|e^{i t \Delta} u_{0}\right\|_{L_{t}^{q} L_{x}^{r}\left(\mathbb{R} \times \mathbb{R}^{d}\right)} \lesssim\left\|u_{0}\right\|_{L^{2}\left(\mathbb{R}^{d}\right)},
$$

where $(q, r)$ is any Schrödinger admissible pair, i.e., $2 \leqslant q, r \leqslant \infty, 2 / q+d / r=d / 2$, and $(q, r, d) \neq$ $(2, \infty, 2)$. The implicit constants depend on $(q, r, d)$. These estimates are of fundamental importance in proving both local and global results for nonlinear Schrödinger equations. (See [Tao 2006; Keel and Tao 1998].)

In the case of compact manifolds, the first Strichartz estimates were proved by Bourgain [1993] in the case of the torus. The case of general compact Riemannian manifolds $(M, g)$ without boundary was dealt with by Burq, Gerard, and Tzvetkov in [Burq et al. 2004] and [Staffilani and Tataru 2002]. In [Burq et al. 2004], the authors prove the estimates

$$
\left\|e^{i t \Delta_{g}} u_{0}\right\|_{L_{t}^{q} L_{x}^{r}[[0,1] \times M)} \lesssim q, r, M\left\|u_{0}\right\|_{H^{1 / q}(M)}
$$

for any admissible pair $(q, r)$. The proof relies on a construction of an approximate parametrix to the semiclassical operator $e^{i h \Delta_{g}} \varphi\left(h \sqrt{-\Delta_{g}}\right)$ (where $\varphi$ is Schwartz) which is used to prove the semiclassical linear Strichartz estimate

$$
\left\|e^{i t \Delta_{g}} u_{0}\right\|_{L_{t}^{q} L_{x}^{r}([0, \alpha / N] \times M)} \lesssim q, r, M\left\|u_{0}\right\|_{L^{2}(M)}
$$

whenever $u_{0}$ is frequency (spectrally) localized at the dyadic scale $N$ and $\alpha \ll 1$. This estimate conforms with the heuristic that Schrödinger evolution moves wavepackets localized at frequency $\sim N$ at speeds $\sim N$, which means that in the time interval $[0, \alpha / N]$, one expects the wave packet to remain in a coordinate patch and hence satisfy the same estimates like those on $\mathbb{R}^{d}$. This heuristic will be very useful in predicting the right bilinear estimate later on as well. Notice that (1-8) follows directly from (1-9) by splitting the time interval $[0,1]$ into $N$ subintervals of lengths $N^{-1}$ and using the conservation of mass and a square function estimate (see [Burq et al. 2004]).

Turning to bilinear estimates, we will start by mentioning the relevant estimate on $\mathbb{R}^{d}$ for which we wish to find an analogue on compact manifolds. This estimate first appeared as a refinement to linear Strichartz estimates in [Bourgain 1998]: assuming that $u_{0}$ is frequency localized at frequencies $\left\{\xi \in \mathbb{R}^{d}:|\xi| \sim N_{1}\right\}$ and $v_{0}$ is frequency localized at frequencies $\left\{\xi \in \mathbb{R}^{d}:|\xi| \lesssim N_{2}\right\}$ with $N_{2} \leqslant N_{1}$, then

$$
\left\|e^{i t \Delta} u_{0} e^{i t \Delta} v_{0}\right\|_{L^{2}\left(\mathbb{R} \times \mathbb{R}^{d}\right)} \lesssim_{d} \frac{N_{2}^{(d-1) / 2}}{N_{1}^{1 / 2}}\|u\|_{L^{2}\left(\mathbb{R}^{d}\right)}\|v\|_{L^{2}\left(\mathbb{R}^{d}\right)} .
$$

We first notice that this estimate is an improvement on applying Hölder's inequality and the linear Strichartz estimates. In fact, applying the linear estimates only, one would get instead of the $N_{2}^{(d-1) / 2} / N_{1}^{1 / 2}$ constant on the left side of (1-10): 1 for $d=2$ (here one uses the $L_{x}^{2} \rightarrow L_{t, x}^{4}$ Strichartz estimate) and $N_{2}^{d / 2-1}$ for $d \geqslant 3$ (here one should use Hölder, the $L_{x}^{2} \rightarrow L_{t, x}^{2(d+2) / d}$ estimate for $e^{i t \Delta} u_{0}$, and Bernstein combined 
with the

$$
L_{x}^{2} \rightarrow L_{t}^{d+2} L_{x}^{\frac{2 d(d+2)}{d(d+2)-4}}
$$

estimate for $e^{i t \Delta} v_{0}$ ). Bourgain used this improvement (when $N_{2} \ll N_{1}$ ) to prove, among other things, global well-posedness below energy norm for certain mass (and $\dot{H}^{1 / 2}$ )-critical equations (which incidentally is also an application that will be considered in the context of closed manifolds in [Hani 2012]). Since then, this improvement and variants of it proved to be of essential use in studying nonlinear Schrödinger equations.

In the context of compact manifolds, some bilinear estimates on the torus were already implicit in [Bourgain 1993] (see also [Burq et al. 2005a]), and other variants were proved in [De Silva et al. 2007]. In [Burq et al. 2005a; 2005b], the authors prove bilinear Strichartz estimates on spheres $S^{2}$ and $S^{3}$ (and on the bit wider class of Zoll manifolds) using bilinear eigenfunction cluster estimates. These bilinear Strichartz estimates take the form

$$
\left\|e^{i t \Delta_{g}} u_{0} e^{i t \Delta_{g}} v_{0}\right\|_{L_{t, x}^{2}\left([0,1] \times S^{d}\right)} \lesssim d N_{2}^{\alpha_{d}}\left\|u_{0}\right\|_{L^{2}\left(S^{d}\right)}\left\|v_{0}\right\|_{L^{2}\left(S^{d}\right)}
$$

whenever $u_{0}$ is spectrally localized in the dyadic region $\sqrt{-\Delta_{g}} \in\left[N_{1}, 2 N_{1}\right), v_{0}$ in the region $\sqrt{-\Delta_{g}} \in$ $\left[N_{2}, 2 N_{2}\right), N_{2} \leqslant N_{1}$, with $\alpha=\frac{1}{4}+\epsilon$ when $d=2$ and $\alpha=\frac{1}{2}+\epsilon$ when $d=3$.

Using Theorem 1.1, we will be able to prove the following bilinear estimate for any closed manifold $(M, g)$ :

Theorem 1.2. Suppose $u_{0}, v_{0} \in L^{2}\left(M^{d}\right)$ are spectrally localized at dyadic scales $N_{1}$ and $N_{2}$ as above with $N_{2} \leqslant N_{1}$. Then the estimate

$$
\left\|e^{i t \Delta_{g}} u_{0} e^{i t \Delta_{g}} v_{0}\right\|_{L_{t, x}^{2}\left(\left[-1 / N_{1}, 1 / N_{1}\right] \times M\right)} \lesssim_{M} \frac{N_{2}^{(d-1) / 2}}{N_{1}^{1 / 2}}\left\|u_{0}\right\|_{L^{2}(M)}\left\|v_{0}\right\|_{L^{2}(M)} .
$$

holds. More generally,

$$
\left\|e^{i t \Delta_{g}} u_{0} e^{i t \Delta_{g}} v_{0}\right\|_{L^{2}([-T, T] \times M)} \leqslant \Lambda\left(T, N_{1}, N_{2}\right)\left\|u_{0}\right\|_{L^{2}(M)}\left\|v_{0}\right\|_{L^{2}(M)},
$$

where

$$
\Lambda\left(T, N_{1}, N_{2}\right) \lesssim_{M} \begin{cases}N_{2}^{(d-1) / 2} / N_{1}^{1 / 2} & \text { if } T \ll N_{1}^{-1}, \\ T^{1 / 2} N_{2}^{(d-1) / 2} & \text { if } T \gtrsim N_{1}^{-1}\end{cases}
$$

In particular, for $T=1$ we have

$$
\left\|e^{i t \Delta_{g}} u_{0} e^{i t \Delta_{g}} v_{0}\right\|_{L^{2}([-1,1] \times M)} \lesssim N_{2}^{(d-1) / 2}\left\|u_{0}\right\|_{L^{2}(M)}\left\|v_{0}\right\|_{L^{2}(M)} .
$$

Some notes are in order: First we notice that in the semiclassical/ short-range case (1-11), the coefficient $N_{2}^{(d-1) / 2} / N_{1}^{1 / 2}$ is the same as that on $\mathbb{R}^{d}$. This conforms with the heuristic that in the time interval $\left[0,1 / N_{1}\right]$, the two waves $e^{i t \Delta_{g}} v_{0}$ (which is moving with speed $\sim N_{1}$ ) and $e^{i t \Delta_{g}} v_{0}$ (moving at speed $\sim N_{2} \leqslant N_{1}$ ) do not leave a coordinate patch and hence their product satisfies the same estimate as that on $\mathbb{R}^{d}$. Second, the estimates in (1-12) and (1-14) are essentially obtained from (1-11) by splitting the time interval into pieces of length $N_{1}^{-1}$. It should be emphasized though that the exact dependence of 
$\Lambda\left(T, N_{1}, N_{2}\right)$ on its all parameters is often of great importance in applications (see [Hani 2012]). In fact, it is easy to see that bilinear estimates on the interval $[0, T]$ translate by scaling into bilinear estimates on the interval $[0,1]$ for the rescaled manifold $\lambda M .^{2}$ The $\lambda$-dependence of those estimates is dictated by dependence of $\Lambda\left(T, N_{1}, N_{2}\right)$ on all its parameters. The bilinear Strichartz estimates on $\lambda M$ take the following form (see [Hani 2012] for relevant calculations):

Corollary 1.3 (Time $T$ estimate on $M$ implies time 1 estimate on $\lambda M$ ). Let $M$ be a $2 D$ closed manifold and suppose that $N_{1}, N_{2} \in 2^{\mathbb{Z}}$ and suppose $u_{0}, v_{0} \in L^{2}(\lambda M)$ are spectrally localized around $N_{1}$ and $N_{2}$ respectively, with $N_{2} \leqslant N_{1}$. Then

$$
\begin{aligned}
\left\|e^{i t \Delta_{\lambda}} u_{0} e^{i t \Delta_{\lambda}} v_{0}\right\|_{L^{2}([0,1] \times \lambda M)} & \lesssim_{M} \Lambda\left(\lambda^{-2}, \lambda N_{1}, \lambda N_{2}\right)\left\|u_{0}\right\|_{L^{2}(\lambda M)}\left\|v_{0}\right\|_{L^{2}(\lambda M)} \\
& \lesssim_{M} \begin{cases}\left(N_{2} / N_{1}\right)^{1 / 2}\left\|u_{0}\right\|_{L^{2}(\lambda M)}\left\|v_{0}\right\|_{L^{2}(\lambda M)} & \text { if } \lambda \gg N_{1}, \\
\left(N_{2} / \lambda\right)^{1 / 2}\left\|u_{0}\right\|_{L^{2}(\lambda M)}\left\|v_{0}\right\|_{L^{2}(\lambda M)} & \text { if } \lambda \lesssim N_{1},\end{cases}
\end{aligned}
$$

where we have denoted by $\Delta_{\lambda}$ the Laplace-Beltrami operator on the rescaled manifold $\lambda M$.

Having favorable bounds (in terms of $\lambda$ and $N_{2}$ ) on the right hand side of (1-16) is crucial to obtaining global well-posedness of some nonlinear equations on $M$ below energy norm. In fact, in [Hani 2012] it is proven that the cubic nonlinear Schrödinger equation is globally well-posed in $H^{s}(M)$ for any closed $2 D$ surface $M^{2}$ and all $s>\frac{2}{3}$, a result which matches the current (to the best of our knowledge) minimum regularity needed for global well-posedness on the 2-torus.

Finally, we note that as in the case of bilinear estimates on $\mathbb{R}^{d}$, the bilinear estimates in (1-11) and (1-12) offer a refinement to those obtained by using linear estimates alone. However, this refinement is only visible when one looks at estimates over time intervals $[0, T]$ for $T \ll N_{2}^{-1}$ (or alternatively, estimates on rescaled manifolds). For example, for $d \geqslant 3$, applying Hölder's inequality, the $L_{t}^{\infty} L_{x}^{2}$ bound on $e^{i t \Delta} u_{0}$, Bernstein and the $L_{t}^{2} L_{x}^{2 d /(d-2)}$ for $e^{i t \Delta} v_{0}$, one gets

$$
\left\|e^{i t \Delta} u_{0} e^{i t \Delta} v_{0}\right\|_{L_{t, x}^{2}([0, T] \times M)} \lesssim C\left(T, N_{2}\right)\left\|u_{0}\right\|_{L^{2}(M)}\left\|v_{0}\right\|_{L^{2}(M)},
$$

where $C\left(T, N_{2}\right)=N_{2}^{(d-2) / 2}=N_{2}^{(d-1) / 2} / N_{2}^{1 / 2}$ for $T \lesssim N_{2}^{-1}$ and $C\left(T, N_{2}\right)=T^{1 / 2} N_{2}^{(d-1) / 2}$ for $T \geqslant N_{2}^{-1}$. This shows the improvement offered by (1-12) in the range $T \ll N_{2}^{-1}$ (especially when dealing with low-high frequency interaction $N_{2} \ll N_{1}$ ). This improvement is due to the cancellation happening when we multiply the high frequency wave with the low frequency one. This cancellation is completely ignored by linear estimates. In the case, $d=2$, one would need to prove an estimate for the inadmissible pair $(q, r)=(2, \infty)$. This is possible with an $N^{\epsilon}$ loss. See [Jiang 2011]. In this case, the bilinear estimate (1-12) not only offers a refinement to linear estimates at time scales $T \ll 1$ and in the range $N_{2} \ll N_{1}$, but also yields better estimates in the time scale $T=1$ (no $N_{2}^{\epsilon}$ loss in (1-14)). See [Hani 2012] for details.

The paper is organized as follows. In Section 2 we provide the proof of Theorem 1.1. In Section 3, we review the needed facts about the parametrix construction in [Burq et al. 2004] and prove Theorem 1.2.

\footnotetext{
${ }^{2}$ Here $\lambda M$ can either be viewed as the Riemmannian manifold $\left(M,\left(1 / \lambda^{2}\right) g\right)$ or by embedding $M$ into some ambient space $\mathbb{R}^{N}$ and then applying a dilation by $\lambda$ to get $\lambda M$.
} 
Finally in Section 4 we prove inhomogeneous versions of the bilinear Strichartz estimates stated above in addition to mixed type bilinear estimates for products of the Schrödinger propagator $e^{i t \Delta} u_{0}$ and the half wave propagators $e^{ \pm i t|\nabla| v_{0}}$. These estimates can also be deduced from Theorem 1.1 and have potential applications (to be investigated elsewhere) in studying Zakharov type systems on closed manifolds. We use the notation $A \lesssim B$ to denote $A \leqslant C B$ for some $C>0$ and $A \sim B$ to denote $A \lesssim B \lesssim A$.

\section{Proof of Theorem 1.1}

All implicit constants are allowed to depend on $d, \delta$ and uniform bounds on a finite number of derivatives of $\phi, \psi, a$ and $b$. We have

$$
T_{\lambda} f(t, x) \tilde{T}_{\mu} g(t, x)=\int_{\mathbb{R}^{d}} \int_{\mathbb{R}^{d}} e^{i\left(\lambda \phi\left(t, x, \xi_{1}\right)+\mu \psi\left(t, x, \xi_{2}\right)\right)} a\left(t, x, \xi_{1}\right) b\left(t, x, \xi_{2}\right) f\left(\xi_{1}\right) g\left(\xi_{2}\right) d \xi_{1} d \xi_{2} .
$$

Since the supports of $a$ and $b$ are compact, one can use a finite partition of unity to split $a$ and $b$ into finitely many pieces so that on the support of each piece there exists $t_{0}, x_{0}, \xi_{0}, \xi_{2,0}$ such that

$$
\left|t-t_{0}\right|,\left|x-x_{0}\right|,\left|\xi_{1}-\xi_{0}\right|,\left|\xi_{2}-\xi_{2,0}\right| \leqslant \frac{1}{C}
$$

where $C$ is some large constant depending only on $\delta$ and the uniform norms of $\phi$ and $\psi$ and their derivatives on the compact supports of $a$ and $b$.

Also notice that by applying a rotation $L$ of the domain $\mathbb{R} \times \mathbb{R}^{d}:(t, x)=L^{T}(s, y)$, the left hand side of (1-5) is unaffected, whereas the hypersurfaces $S_{\phi}$ and $S_{\psi}$ are both rotated by $L$. In fact, since

$$
\nabla_{(s, y)}\left(\phi\left(L^{T}(s, y), x, \xi\right)\right)=L(\nabla \phi)\left(L^{T}(s, y), \xi\right)
$$

where $\nabla$ is taken in the first $d+1$ variables of $\phi$. Consequently, if we apply the change of variable $(t, x)=L^{T}(s, y)$, the canonical hypersurfaces $S_{\phi}$ and $S_{\psi}$ are both rotated by $L$. Using this symmetry, one can assume that

$$
\left|\operatorname{det}\left(\frac{\partial^{2} \phi}{\partial \xi \partial x}\left(t_{0}, x_{0}, \xi_{0}\right)\right)\right| \gtrsim 1 \quad \text { and } \quad\left|\operatorname{det}\left(\frac{\partial^{2} \psi}{\partial \xi \partial x}\left(t_{0}, x_{0}, \xi_{2,0}\right)\right)\right| \gtrsim 1
$$

on the support of $a$ and of $b$, respectively. This means that the surfaces $S_{\phi}$ and $S_{\psi}$ can be regarded as graphs of functions of the form $\left(\xi, \tau_{1}(\xi)\right)$ and $\left(\xi, \tau_{2}(\xi)\right) \subset T_{\left(t_{0}, x_{0}\right)}^{*} \mathbb{R}^{d+1}$ respectively.

Define

$$
A:=\frac{\partial^{2} \phi}{\partial \xi \partial x}\left(t_{0}, x_{0}, \xi_{0}\right) \quad \text { and } \quad B:=\frac{\partial^{2} \psi}{\partial \xi \partial x}\left(t_{0}, x_{0}, \xi_{2,0}\right)
$$

By the above, we have that $A$ and $B$ are invertible. It will be convenient later on to do following change of variables in the $\xi_{1}$ integral and define $\xi=\xi_{1}+(\mu / \lambda) A^{-1} B \xi_{2}{ }^{3}$ This gives

$$
\begin{aligned}
& T_{\lambda} f(t, x) \tilde{T}_{\mu} g(t, x) \\
& \quad=\int_{\mathbb{R}^{d}} \int_{\mathbb{R}^{d}} e^{i \lambda\left(\phi\left(t, x, \xi-(\mu / \lambda) A^{-1} B \xi_{2}\right)+(\mu / \lambda) \psi\left(t, x, \xi_{2}\right)\right)} c\left(t, x, \xi, \xi_{2}\right) f\left(\xi-\frac{\mu}{\lambda} A^{-1} B \xi_{2}\right) g\left(\xi_{2}\right) d \xi d \xi_{2},
\end{aligned}
$$

\footnotetext{
${ }^{3}$ The justification for this change of variables will be obvious later on. However, at a heuristic level this corresponds to adding the momenta of the two waves.
} 
where we set $c\left(t, x, \xi, \xi_{2}\right)=a\left(t, x, \xi-(\mu / \lambda) A^{-1} B \xi_{2}\right) b\left(t, x, \xi_{2}\right)$ and all we have to remember about $c$ is that it is uniformly bounded along with all its derivatives (since $\mu / \lambda \leqslant 1$ ) and is supported in a small neighborhood of $\left(t_{0}, x_{0}, \xi_{0}+(\mu / \lambda) A^{-1} B \xi_{2,0}, \xi_{2,0}\right)$ of diameter $\lesssim 1 / C$. In particular, we have

$$
\left|\xi-\frac{\mu}{\lambda} A^{-1} B \xi_{2}-\xi_{0}\right| \leqslant \frac{1}{C}
$$

for every $\xi, \xi_{2}$ in the support of $c$.

We now fix a particular coordinate direction $e_{j}$ (to be specified later), and write $\xi_{2}=p e_{j}+\xi_{2}^{\prime}$. Roughly speaking, the direction will be chosen using the transversality assumption of the two surfaces $S_{\phi}$ and $S_{\psi}$ so that

$$
\left|\left\langle v_{1}\left(\xi_{0}\right), \frac{\partial^{2} \psi\left(t_{0}, x_{0}, \xi_{2,0}\right)}{\partial \xi \partial(t, x)} e_{j}\right\rangle\right| \gtrsim_{\delta} 1 .
$$

(The inner product is in $\mathbb{R}^{d+1}$, the second entry being the product of a $(d+1) \times d$ matrix with a vector in $\mathbb{R}^{d}$.) This will be possible because $v_{2}$ is the unique direction for which

$$
\left\langle v_{2}, \frac{\partial^{2} \psi\left(t_{0}, x_{0}, \xi_{2,0}\right)}{\partial \xi \partial(t, x)}\right\rangle=\overrightarrow{0}_{\mathbb{R}^{d}}
$$

since $v_{1}$ is not a multiple of $v_{2}$, the vector

$$
\left\langle v_{1}, \frac{\partial^{2} \psi\left(t_{0}, x_{0}, \xi_{2,0}\right)}{\partial \xi \partial(t, x)}\right\rangle
$$

is also nonzero, so there exists a coordinate direction $e_{j}$ onto which the projection of this nonzero vector does not vanish. In other words, the inner product in (2-5) can be thought of as the projection of $v_{1}$ onto the curve in $S_{\phi}\left(t_{0}, x_{0}\right)$ given by $t \mapsto \nabla_{(t, x)} \psi\left(t_{0}, x_{0}, \xi_{2,0}+t e_{j}\right)$.

For convenience of notation, when confusion does not arise, we will assume that $j=1$ and write $\xi_{2}=\left(p, \xi_{2}^{\prime}\right)$ where $p \in \mathbb{R}$ and $\xi_{2}^{\prime} \in \mathbb{R}^{d-1}$. As a result, we have

$$
\begin{aligned}
& \left\|T_{\lambda} f(t, x) \tilde{T}_{\mu} g(t, x)\right\|_{2} \\
& \quad=\left\|\int_{\mathbb{R}_{\xi^{\prime}}^{d-1}} \int_{\mathbb{R}_{\xi}^{d}} \int_{\mathbb{R}_{p}} e^{i \lambda\left(\phi\left(t, x, \xi-(\mu / \lambda) A^{-1} B \xi_{2}\right)+(\mu / \lambda) \psi\left(t, x, \xi_{2}\right)\right)} c\left(t, x, \xi, \xi_{2}\right) f\left(\xi-\frac{\mu}{\lambda} \xi_{2}\right) g\left(\xi_{2}\right) d \xi d p d \xi_{2}^{\prime}\right\|_{2} \\
& \quad \leqslant \int_{\mathbb{R}_{\xi_{2}}^{d-1}}\left\|\int_{\mathbb{R}_{\xi}^{d}} \int_{\mathbb{R}_{p}} e^{i \lambda\left(\phi\left(t, x, \xi-(\mu / \lambda) A^{-1} B \xi_{2}\right)+(\mu / \lambda) \psi\left(t, x, \xi_{2}\right)\right)} c\left(t, x, \xi, \xi_{2}\right) f\left(\xi-\frac{\mu}{\lambda} \xi_{2}\right) g\left(\xi_{2}\right) d \xi d p\right\|_{L_{t, x}^{2}} d \xi_{2}^{\prime} .
\end{aligned}
$$

Freezing $\xi_{2}^{\prime}$, we define the operator $S=S_{\xi_{2}^{\prime}}: L^{2}\left(\mathbb{R}^{d+1}\right) \rightarrow L^{2}\left(\mathbb{R}^{d+1}\right)$ given by

$$
S F(t, x)=\int_{\mathbb{R}_{\xi}^{d}} \int_{\mathbb{R}_{p}} e^{i \lambda\left(\phi\left(t, x, \xi-(\mu / \lambda) A^{-1} B \xi_{2}\right)+(\mu / \lambda) \psi\left(t, x, \xi_{2}\right)\right)} c\left(t, x, \xi, \xi_{2}\right) F(\xi, p) d \xi d p,
$$

where $\xi_{2}=\left(p, \xi_{2}^{\prime}\right)$. As a result of this definition, our estimate is reduced to proving that for each $\xi_{2}^{\prime}$, the estimate

$$
\|S F\|_{L_{t, x}^{2}\left(\mathbb{R}^{d+1}\right)} \lesssim \frac{1}{\lambda^{d / 2} \mu^{1 / 2}}\|F\|_{L_{p, \xi}^{2}\left(\mathbb{R}^{d+1}\right)}
$$


holds for $S$. In fact, with such an estimate and by Cauchy-Schwarz in the $\xi_{2}^{\prime}$ integral (keeping in mind that $c$ is compactly supported), we get

$$
\begin{aligned}
\left\|T_{\lambda} f(t, x) \tilde{T}_{\mu} g(t, x)\right\|_{2} & \lesssim \frac{1}{\lambda^{d / 2} \mu^{1 / 2}} \int_{\left|\xi_{2}^{\prime}\right| \lesssim 1}\left\|f\left(\xi-\frac{\mu}{\lambda}\left(p, \xi_{2}^{\prime}\right)\right) g\left(p, \xi_{2}^{\prime}\right)\right\|_{L_{p, \xi}^{2}} d \xi_{2}^{\prime} \\
& \lesssim \frac{1}{\lambda^{d / 2} \mu^{1 / 2}}\|f\|_{L^{2}}\|g\|_{L^{2}}
\end{aligned}
$$

The bound on $S$ is proved using a $T^{*} T$ argument. For convenience of notation, let us define

$$
\Phi(t, x, \xi, p)=\phi\left(t, x, \xi-\frac{\mu}{\lambda} A^{-1} B \xi_{2}\right)+\frac{\mu}{\lambda} \psi\left(t, x, \xi_{2}\right)
$$

where $\xi_{2}=\left(p, \xi_{2}^{\prime}\right)$. With this notation, $S$ takes the form

$$
S F(t, x)=\int_{\mathbb{R}_{\xi}^{d}} \int_{\mathbb{R}_{p}} e^{i \lambda \Phi(t, x, \xi, p)} c(t, x, \xi, p) F(\xi, p) d \xi d p
$$

The adjoint of $S$ is given by the operator

$$
S^{*} G(\xi, p)=\int_{\mathbb{R}_{x}^{d}} \int_{\mathbb{R}_{t}} e^{-i \lambda \Phi(t, x, \xi, p)} \bar{c}(t, x, \xi, p) G(x, t) d x d t .
$$

As a result, we get

$$
S^{*} S F(\zeta, q)=\int_{\mathbb{R}_{\xi}^{d}} \int_{\mathbb{R}_{p}} K(\zeta, q, \xi, p) F(\xi, p) d \xi d p
$$

where

$$
K(\zeta, q, \xi, p)=\int_{\mathbb{R}_{t}} \int_{\mathbb{R}_{x}^{d}} e^{i \lambda[\Phi(t, x, \xi, p)-\Phi(t, x, \zeta, q)]} c(t, x, \xi, p) \bar{c}(t, x, \zeta, q) d x d t
$$

Our aim will be to show that $K$ satisfies the bound

$$
K(\zeta, q, \xi, p) \lesssim_{N} \frac{1}{(1+\lambda|\xi-\zeta|+\mu|q-p|)^{N}}
$$

for a sufficiently large $N$ (any $N>d+1$ would do).

In fact, with such an estimate, one can easily see (using Schur's test for example) that $\left\|S^{*} S\right\|_{L^{2} \rightarrow L^{2}} \lesssim$ $1 /\left(\lambda^{d} \mu\right)$. Since $\|S\|_{L^{2} \rightarrow L^{2}}=\left\|S^{*} S\right\|_{L^{2} \rightarrow L^{2}}^{1 / 2}$ one gets that $\|S\|_{L^{2} \rightarrow L^{2}}$ is bounded by $O\left(1 /\left(\lambda^{d / 2} \mu^{1 / 2}\right)\right)$.

The bound on $K$ is based on nonstationary-phase-type estimates and integration by parts. These are based on the following estimates on the phase function $\Phi$ and its derivatives.

Lemma 2.1. There exists $\Omega \in S^{d}$ such that

$$
\mid\left\langle\nabla_{t, x} \Phi(t, x, \xi, p)-\nabla_{t, x} \Phi(t, x, \zeta, q), \Omega|| \gtrsim|\xi-\zeta|+\frac{\mu}{\lambda}|p-q|\right.
$$

and

$$
\left|\frac{\partial}{\partial x^{\alpha} \partial t^{\beta}}(\Phi(t, x, \xi, p)-\Phi(t, x, \zeta, q))\right| \lesssim_{\alpha, \beta}|\xi-\zeta|+\frac{\mu}{\lambda}|p-q|
$$


Proof. The second estimate (2-13) is a direct consequence of the definition (2-8), the Taylor expansion, and the uniform boundedness of all the $t, x$ derivatives of $\phi$ and $\psi$. We now turn to the proof of (2-12).

Here we split the analysis into two cases:

Case 1: $|\xi-\zeta| \geqslant \frac{1}{100}(\mu / \lambda)|p-q|$. The change of variables we have made in (2-3) will allow us to prove (2-12) in this case using only the $x$ derivative part of $\nabla_{t, x} \Phi$. In fact, using (2-8), we have

$$
\begin{aligned}
\nabla_{x} \Phi(t, x, \xi, p)-\nabla_{x} \Phi(t, x, \zeta, q)= & \nabla_{x} \phi\left(t, x, \xi-\frac{\mu}{\lambda} A^{-1} B \xi_{2}\right)-\nabla_{x} \phi\left(t, x, \zeta-\frac{\mu}{\lambda} A^{-1} B \zeta_{2}\right) \\
& +\frac{\mu}{\lambda}\left(\nabla_{x} \psi\left(t, x, \xi_{2}\right)-\nabla_{x} \psi\left(t, x, \zeta_{2}\right)\right),
\end{aligned}
$$

where $\zeta_{2}=\left(q, \xi_{2}^{\prime}\right)$. We estimate (2-14) in the following manner:

$$
\begin{aligned}
\nabla_{x} \phi\left(t, x, \xi-\frac{\mu}{\lambda} A^{-1} B \xi_{2}\right)- & \nabla_{x} \phi\left(t, x, \zeta-\frac{\mu}{\lambda} A^{-1} B \zeta_{2}\right) \\
& =\left\langle\frac{\partial^{2} \phi}{\partial \xi \partial x}\left(t, x, \xi-\frac{\mu}{\lambda} A^{-1} B \xi_{2}\right), \xi-\zeta-\frac{\mu}{\lambda} A^{-1} B\left(\xi_{2}-\zeta_{2}\right)\right\rangle+O\left(|\xi-\zeta|^{2}\right) \\
& =\left\langle\frac{\partial^{2} \phi}{\partial \xi \partial x}\left(t_{0}, x_{0}, \xi_{0}\right), \xi-\zeta-\frac{\mu}{\lambda} A^{-1} B\left(\xi_{2}-\zeta_{2}\right)\right\rangle+\text { Error }_{1} \\
& =A(\xi-\zeta)-\frac{\mu}{\lambda} B\left(\xi_{2}-\zeta_{2}\right)+\text { Error }_{1},
\end{aligned}
$$

where we used the fact that $A=\left(\partial^{2} \phi / \partial \xi \partial x\right)\left(t_{0}, x_{0}, \xi_{0}\right)$. Here

$$
\begin{aligned}
\text { Error }_{1}=\left\langle\frac{\partial^{2} \phi}{\partial \xi \partial x}\left(t, x, \xi-\frac{\mu}{\lambda} A^{-1} B \xi_{2}\right),\right. & \left.\xi-\zeta-\frac{\mu}{\lambda} A^{-1} B\left(\xi_{2}-\zeta_{2}\right)\right\rangle \\
& -\left\langle\frac{\partial^{2} \phi}{\partial \xi \partial x}\left(t_{0}, x_{0}, \xi_{0}\right), \xi-\zeta-\frac{\mu}{\lambda} A^{-1} B\left(\xi_{2}-\zeta_{2}\right)\right\rangle+O\left(|\xi-\zeta|^{2}\right) .
\end{aligned}
$$

By our assumption of smallness of the support of $c$ (cf. (2-4)), the error can be estimated (if $C$ is chosen large enough depending on the uniform norms of derivatives of $\phi$ ) by

$$
\mid \text { Error }_{1}\left|\lesssim_{\phi} \frac{1}{C}\right| \xi-\zeta-\frac{\mu}{\lambda} A^{-1} B\left(\zeta_{2}-\xi_{2}\right)\left|+O\left(|\xi-\zeta|^{2}\right) \leqslant \frac{1}{10} \gamma_{1}\right| \xi-\zeta \mid
$$

where $\gamma_{1}$ is chosen to be the smallest singular value of $A$ (or equivalently $\gamma_{1}=\min _{z \in S^{d-1}}|A z|$ ).

Next we estimate (2-15) by

$$
\begin{aligned}
\frac{\mu}{\lambda}\left(\nabla_{x} \psi\left(t, x, \xi_{2}\right)-\nabla_{x} \psi\left(t, x, \zeta_{2}\right)\right) & =\frac{\mu}{\lambda}\left\langle\frac{\partial^{2} \psi}{\partial \xi \partial x}\left(t, x, \xi_{2}\right), \xi_{2}-\zeta_{2}\right\rangle+O\left(\frac{\mu}{\lambda}\left|\xi_{2}-\zeta_{2}\right|^{2}\right) \\
& =\frac{\mu}{\lambda}\left\langle\frac{\partial^{2} \psi}{\partial \xi \partial x}\left(t_{0}, x_{0}, \xi_{2,0}\right), \xi_{2}-\zeta_{2}\right\rangle+\text { Error }_{2} \\
& =\frac{\mu}{\lambda} B\left(\xi_{2}-\zeta_{2}\right)+\text { Error }_{2},
\end{aligned}
$$

where

$$
\text { Error }_{2}=\frac{\mu}{\lambda}\left(\left\langle\frac{\partial^{2} \psi}{\partial \xi \partial x}\left(t, x, \xi_{2}\right), \xi_{2}-\zeta_{2}\right\rangle-\left\langle\frac{\partial^{2} \psi}{\partial \xi \partial x}\left(t_{0}, x_{0}, \xi_{2,0}\right), \xi_{2}-\zeta_{2}\right\rangle\right)+O\left(\frac{\mu}{\lambda}\left|\xi_{2}-\zeta_{2}\right|^{2}\right)
$$


which, as before, can be bounded (using the bounds $\left|\xi_{2}-\zeta_{2}\right|,\left|\xi_{2}-\xi_{2,0}\right| \lesssim \frac{1}{C}$ and $\frac{\mu}{\lambda}\left|\xi_{2}-\zeta_{2}\right| \leqslant 100|\xi-\zeta|$ ) by

$$
\mid \text { Error }_{2}\left|\leqslant \frac{1}{10} \gamma_{1}\right| \xi-\zeta \mid
$$

Collecting these estimates we get

$$
\nabla_{x} \Phi(t, x, \xi, p)-\nabla_{x} \Phi(t, x, \zeta, q)=A(\zeta-\xi)+\text { Error }_{1}+\text { Error }_{2},
$$

where Error ${ }_{1}+$ Error $_{2}$ is bounded by $\frac{1}{5} \gamma_{1}|\zeta-\xi|$. We now let $\omega \in S^{d-1}$ be equal to $A(\zeta-\xi) /|A(\zeta-\xi)|$. Since

$$
|\langle A(\zeta-\xi), \omega\rangle|=|A(\xi-\zeta)| \geqslant \gamma_{1}|\xi-\zeta|
$$

by the definition of $\gamma_{1}$, we get

$$
\left|\left\langle\nabla_{x} \Phi(t, x, \xi, p)-\nabla_{x} \Phi(t, x, \zeta, q), \omega\right\rangle\right| \gtrsim|\xi-\zeta| .
$$

As a result, by taking $\Omega \in S^{d}$ equal to $(\omega, 0)$ we get

$$
\left|\left\langle\nabla_{t, x} \Phi(t, x, \xi, p)-\nabla_{t, x} \Phi(t, x, \zeta, q), \Omega\right\rangle\right| \gtrsim|\xi-\zeta| \gtrsim|\xi-\zeta|+\frac{\mu}{\lambda}|p-q|,
$$

which is (2-12) in Case 1.

Case 2: $|\xi-\zeta| \leqslant \frac{1}{100}(\mu / \lambda)|p-q|$. The analysis in this case is a bit more delicate, and it is here that the transversality assumption is used. In this case, we will take $\Omega=v_{1}\left(\xi_{0}\right)$, the normal to the surface $\xi \mapsto \nabla_{t, x} \phi\left(t_{0}, x_{0}, \xi\right)$ at $\xi_{0}$. With this choice we have

$\left\langle\nabla_{t, x} \Phi(t, x, \xi, p)-\nabla_{t, x} \Phi(t, x, \zeta, q), \Omega\right\rangle$

$$
\begin{aligned}
= & \left\langle\nabla_{t, x} \phi\left(t, x, \xi-\frac{\mu}{\lambda} A^{-1} B \xi_{2}\right)-\nabla_{t, x} \phi\left(t, x, \zeta-\frac{\mu}{\lambda} A^{-1} B \zeta_{2}\right), v_{1}\left(\xi_{0}\right)\right\rangle \\
& +\frac{\mu}{\lambda}\left\langle\nabla_{t, x} \psi\left(t, x, \xi_{2}\right)-\nabla_{t, x} \psi\left(t, x, \zeta_{2}\right), v_{1}\left(\xi_{0}\right)\right\rangle .
\end{aligned}
$$

The main term in this expression comes from (2-19), whereas (2-18) will be treated as an error. We start by lower bounding (2-19).

We have

$$
\begin{aligned}
\nabla_{t, x} \psi\left(t, x, \xi_{2}\right)-\nabla_{t, x} \psi\left(t, x, \zeta_{2}\right) & =\left\langle\frac{\partial^{2} \psi}{\partial \xi \partial(x, t)}\left(t, x, \xi_{2}\right), \xi_{2}-\zeta_{2}\right\rangle+O\left(\left|\xi_{2}-\zeta_{2}\right|^{2}\right) \\
& =\left\langle\frac{\partial^{2} \psi}{\partial \xi \partial(x, t)}\left(t_{0}, x_{0}, \xi_{2,0}\right), \xi_{2}-\zeta_{2}\right\rangle+\text { Error }_{1} \\
& =(p-q)\left\langle\frac{\partial^{2} \psi}{\partial \xi \partial(x, t)}\left(t_{0}, x_{0}, \xi_{2,0}\right), e_{j}\right\rangle+\text { Error }_{1}
\end{aligned}
$$

where

$$
\text { Error }_{1}=\left\langle\frac{\partial^{2} \psi}{\partial \xi \partial(x, t)}\left(t, x, \xi_{2}\right), \xi_{2}-\zeta_{2}\right\rangle-\left\langle\frac{\partial^{2} \psi}{\partial \xi \partial(x, t)}\left(t_{0}, x_{0}, \xi_{2,0}\right), \xi_{2}-\zeta_{2}\right\rangle+O\left(\left|\xi_{2}-\zeta_{2}\right|^{2}\right)
$$


This is estimated as before using the small support assumption to get

$$
\mid \text { Error }_{1}\left|\lesssim \psi \frac{1}{C}\right| \xi_{2}-\zeta_{2}\left|\leqslant \frac{1}{C}\right| p-q \mid,
$$

where we have used in the last inequality the fact that $\xi_{2}=\left(p, \xi_{2}^{\prime}\right)$ and $\zeta_{2}=\left(q, \xi_{2}^{\prime}\right)$. We remark that

$$
N:=\frac{\partial^{2} \psi}{\partial \xi \partial(x, t)}\left(t_{0}, x_{0}, \xi_{2,0}\right)
$$

is a $(d+1) \times d$ matrix, so $\left\langle N, e_{j}\right\rangle$ is a vector in $\mathbb{R}^{d+1}$. From a geometric point of view, this vector lies in the tangent space to $S_{\psi}\left(t_{0}, x_{0}\right)$ at $\xi_{2,0}$.

Recall that by definition, $v_{2}:=v_{2}\left(\xi_{2,0}\right)$ is the unique vector (up to sign) in $S^{d}$ such that $v_{2}^{T} N=0$ where $v_{2}^{T}$ is the row vector corresponding to $v_{2}$. In particular, the map from the $d$-dimensional subspace $\nu_{2}^{\perp} \subset \mathbb{R}^{d+1}$ into $\mathbb{R}^{d}$ given by

$$
v \in v_{2}^{\perp} \mapsto v^{T} N \in \mathbb{R}^{d}
$$

is an isomorphism. Let $\gamma_{2}>0$ denote its smallest singular value (or equivalently $\gamma_{2}$ is the positive infimum of the above map when $v \in v_{2}^{\perp}$ satisfies $\|v\|=1$ ).

Writing $v_{1}\left(\xi_{0}\right)=\alpha v_{2}+\beta \nu_{3}$ with $v_{3} \in v_{2}^{\perp},\left\|\nu_{3}\right\|=1$, and $|\alpha|,|\beta| \leqslant 1$, we notice that since $1-\delta>$ $\left|\left\langle v_{1}, v_{2}\right\rangle\right|=|\alpha|$ we have that $|\beta|=\sqrt{1-\alpha^{2}} \geqslant \sqrt{\delta}$.

As a result, we have

$$
\left\langle v_{1}, \nabla_{t, x} \psi\left(t, x, \xi_{2}\right)-\nabla_{t, x} \psi\left(t, x, \zeta_{2}\right)\right\rangle=(p-q) v_{1}^{T} N e_{j}+\text { Error }_{1}=\beta(p-q) v_{3}^{T} N e_{j}+\text { Error }_{1} .
$$

Since $\left\|v_{3}^{T} N\right\| \geqslant \gamma_{2}$, one can choose $e_{j}$ so that $\left|v_{3}^{T} N e_{j}\right| \geqslant \gamma_{2} / \sqrt{d}=: c_{1}$. Combining this to the estimate on Error 1 in (2-20) above we get that if $C$ is large enough, then

$$
\mid\left\langle v_{1}, \nabla_{t, x} \psi\left(t, x, \xi_{2}\right)-\nabla_{t, x} \psi\left(t, x, \zeta_{2}\right)|| \geqslant c_{1} \sqrt{\delta}|p-q|-\frac{c_{1} \sqrt{\delta}}{100}|p-q| \geqslant \frac{99}{100} c_{1} \sqrt{\delta}|p-q| .\right.
$$

As mentioned before, we will treat (2-18) as an error. Indeed,

$$
\begin{aligned}
\left\langle\nabla_{t, x} \phi(t, x, \xi\right. & \left.\left.-\frac{\mu}{\lambda} A^{-1} B \xi_{2}\right)-\nabla_{t, x} \phi\left(t, x, \zeta-\frac{\mu}{\lambda} A^{-1} B \zeta_{2}\right), v_{1}\left(\xi_{0}\right)\right\rangle \\
& =v_{1}\left(\xi_{0}\right)^{T} D_{(d+1) \times d}\left(t, x, \xi-\frac{\mu}{\lambda} A^{-1} B \xi_{2}\right)\left[\xi-\zeta-\frac{\mu}{\lambda} A^{-1} B\left(\xi_{2}-\zeta_{2}\right)\right]+O\left(\left|\frac{\mu}{\lambda}(p-q)\right|^{2}\right),
\end{aligned}
$$

where we have defined

$$
D_{(d+1) \times d}(t, x, \eta)=\frac{\partial^{2} \phi}{\partial \xi \partial(x, t)}(t, x, \eta)
$$

and also used that $|\xi-\zeta| \leqslant(\mu / \lambda)|p-q|$ in this case. Since the derivatives of $D$ are uniformly bounded and because of the small support assumption (2-4), we have

$$
\left\|D_{(d+1) \times d}\left(t, x, \xi-\frac{\mu}{\lambda} A^{-1} B \xi_{2}\right)-D_{(d+1) \times d}\left(t_{0}, x_{0}, \xi_{0}\right)\right\| \lesssim \frac{1}{C} \leqslant \frac{c_{1} \sqrt{\delta}}{100\left(\left\|A^{-1} B\right\|+1\right)}
$$

if $C$ is large enough. 
Using the fact that $v_{1}^{T} D_{(d+1) \times d}\left(t_{0}, x_{0}, \xi_{0}\right)=0$, we get that

$$
\left|\left\langle\nabla_{t, x} \phi\left(t, x, \xi-\frac{\mu}{\lambda} A^{-1} B \xi_{2}\right)-\nabla_{t, x} \phi\left(t, x, \zeta-\frac{\mu}{\lambda} A^{-1} B \zeta_{2}\right), v_{1}\left(\xi_{0}\right)\right\rangle\right| \leqslant \frac{c_{1} \sqrt{\delta}}{50} \frac{\mu}{\lambda}|p-q|
$$

again using the small support assumption.

Combining (2-22) and (2-21), we get (2-12) for Case 2.

Now we are ready to perform the integration by parts needed to prove the estimate (2-11). Recall that

$$
K(\zeta, q, \xi, p)=\int_{\mathbb{R}_{t}} \int_{\mathbb{R}_{x}^{d}} e^{i \lambda[\Phi(t, x, \xi, p)-\Phi(t, x, \zeta, q)]} c(t, x, \xi, p) \bar{c}(t, x, \zeta, q) d x d t .
$$

Let $D_{\Omega}$ be the operator given by

$$
D_{\Omega}:=\frac{1}{i \lambda\left\langle\nabla_{t, x} \Phi(t, x, \xi, p)-\nabla_{t, x} \Phi(t, x, \zeta, q), \Omega\right\rangle}\left\langle\nabla_{(x, t)}, \Omega\right\rangle
$$

Then

$$
D_{\Omega}\left(e^{i \lambda\left(\Phi\left(t, x, \xi, \xi_{2}\right)-\Phi\left(t, x, \zeta, \zeta_{2}\right)\right)}\right)=e^{i \lambda\left(\Phi\left(t, x, \xi, \xi_{2}\right)-\Phi\left(t, x, \zeta, \zeta_{2}\right)\right)} .
$$

Noticing that the formal adjoint of $D_{\Omega}$ acting on $L^{2}$ is

$$
D_{\Omega}^{T}=\left\langle\nabla_{(x, t)}, \Omega\right\rangle \frac{1}{\left(i \lambda\left\langle\nabla_{t, x} \bar{\Phi}(t, x, \xi, p)-\nabla_{t, x} \bar{\Phi}(t, x, \zeta, q), \Omega\right\rangle\right)},
$$

we get

$$
\begin{aligned}
K(\zeta, q, \xi, p) & =\int_{\mathbb{R}_{t}} \int_{\mathbb{R}_{x}^{d}} e^{i \lambda[\Phi(t, x, \xi, p)-\Phi(t, x, \zeta, q)]} c(t, x, \xi, p) \bar{c}(t, x, \zeta, q) d x d t \\
& =\int_{\mathbb{R}_{t}} \int_{\mathbb{R}_{x}^{d}} e^{i \lambda[\Phi(t, x, \xi, p)-\Phi(t, x, \zeta, q)]} \overline{\left(D_{\Omega}^{T}\right)^{N} \bar{c}(t, x, \xi, p) c(t, x, \zeta, q)} d x d t
\end{aligned}
$$

Using the estimates in Lemma 2.1, it is easy to see that

$$
\left(D_{\Omega}^{T}\right)^{N} \bar{c}(t, x, \xi, p) c(t, x, \zeta, q) \lesssim_{N} \frac{1}{(\lambda|\xi-\zeta|+\mu|p-q|)^{N}}
$$

When $\lambda|\xi-\zeta|+\mu|p-q| \leqslant 1$, we do not perform any integration by parts and estimate the $K$ integrand by $O(1)$ and hence $K$ by $O(1)$ as well. Otherwise we use the above decay. As a result, we get

$$
K\left(\xi, \xi_{2}, \zeta, \zeta_{2}\right) \lesssim_{N} \frac{1}{(1+\lambda|\xi-\zeta|+\mu|p-q|)^{N}}
$$

which finishes the proof.

Remark. It is not hard to see that the estimate (1-5) is sharp. In fact, by considering the restriction case and taking $\phi(t, x, \xi)=\psi(t, x, \xi)=x . \xi+t|\xi|^{2}$ with $a$ having its $\xi$ support in the region $|\xi| \geqslant 100$ and $b$ having its $\xi$ support near $|\xi| \leqslant 1$, one can reduce the sharpness of (1-5) to that of (1-10) which is known to be sharp. In fact, this can be seen by first reducing to the case when $N_{2}=1$ (again using scaling) and taking $\widehat{u_{0}}$ to be the characteristic function of $\left[N_{1}, N_{1}+N_{1}^{-1}\right] \times[-1,1]^{d-1}$ (hence $\left\|u_{0}\right\|_{L_{x}^{2}} \sim N_{1}^{-1 / 2}$ ); and $\widehat{v_{0}}$ to be the characteristic function of $[-1,1]^{d}$ (hence $\left\|v_{0}\right\|_{L_{x}^{2}} \sim 1$ ). By Plancherel's 
theorem in space and time, we get that the left side of (1-10) is $\gtrsim\left\|\chi_{R_{1}} * \chi_{R_{2}}\right\|_{L^{2}\left(\mathbb{R}^{d+1}\right)}$ where $R_{1}=$ $\left[N_{1}, N_{1}+N_{1}^{-1}\right] \times[0,1]^{d}$ and $R_{2}=[-1,1]^{d+1}$. A direct calculation now shows that $\chi_{R_{1}} * \chi_{R_{2}} \gtrsim\left(1 / N_{1}\right) \chi_{R_{3}}$ where $R_{3}=\left[N_{1}+\frac{1}{4}, N_{1}+\frac{3}{4}\right] \times\left[-\frac{1}{2}, \frac{1}{2}\right]^{d}$ and hence $\left\|\chi_{R_{1}} * \chi_{R_{2}}\right\|_{L^{2}\left(\mathbb{R}^{d+1}\right)} \sim 1 / N_{1}$, which shows that the left side of (1-10) is $\gtrsim\left(1 / N_{1}^{1 / 2}\right)\left\|u_{0}\right\|_{L_{x}^{2}}\left\|v_{0}\right\|_{L_{x}^{2}}$.

\section{Bilinear Strichartz estimates}

We will apply the result of the previous section to get bilinear Strichartz estimates for the free Schrödinger evolution on compact manifolds without boundary. These will be analogues in the variable coefficient case to the estimate (1-10) on $\mathbb{R}^{d}$ with the Euclidean Laplacian which we recall here for convenience

$$
\left\|e^{i t \Delta} u_{0} e^{i t \Delta} v_{0}\right\|_{L^{2}\left(\mathbb{R} \times \mathbb{R}^{d}\right)} \lesssim \frac{N_{2}^{(d-1) / 2}}{N_{1}^{1 / 2}}\|u\|_{L^{2}\left(\mathbb{R}^{d}\right)}\|v\|_{L^{2}\left(\mathbb{R}^{d}\right)},
$$

where $u, v \in L^{2}\left(\mathbb{R}^{d}\right)$ are frequency localized on the dyadic annuli $\left\{\xi \in \mathbb{R}^{d}:|\xi| \in\left[N_{1}, 2 N_{1}\right]\right\}$ and $\left\{\xi \in \mathbb{R}^{d}:|\xi| \in\left[N_{2}, 2 N_{2}\right]\right\}$ respectively.

By scaling time and space, one can easily see that this estimate is equivalent to the same one on the time interval $\left[0,1 / N_{1}\right]$. On this time scale, the numerology in (1-10) can be understood (heuristically at least) by a simple back-of-the-envelope calculation. Thinking of $e^{i t \Delta} u_{0}$ as a "bump function" localized in frequency at scale $N_{1}$ and initially (at $t=0$ ) localized in space at scale $1 / N_{1}$. The evolution moves this bump function at a speed $N_{1}$ thus expanding its support at this rate while keeping the $L^{2}$ norm conserved. Similarly, $e^{i t \Delta} v_{0}$ could be thought of as a "bump function" that is initially concentrated in space at scale $\sim 1 / N_{2}$ and moving (expanding) at speed $N_{2}$. A simple schematic diagram allows to estimate the space-time overlap of the two expanding "bump functions" thus giving the estimate $N_{2}^{(d-1) / 2} / N_{1}^{1 / 2}$ for the $L_{t, x}^{2}\left(\left[0, N_{1}^{-1}\right] \times \mathbb{R}^{d}\right)$ of the product.

The goal of this section is to prove the analogue of (1-10) for the linear evolution of the Schrödinger equation on a $C^{\infty}$ compact manifold $M$ without boundary. This was stated in Theorem 1.2. All implicit constants are allowed to depend on $M$ and the uniform bounds of its metric functions (they are all finite since $M$ is compact). To fix notation, we consider two functions $u_{0}, v_{0} \in C^{\infty}(M)^{4}$ such that $u_{0}=\varphi\left(\sqrt{-\Delta} / N_{1}\right) u_{0}$ and $v_{0}=\varphi\left(\sqrt{-\Delta} / N_{2}\right) v_{0}$ where $\varphi \in C_{0}^{\infty}(\mathbb{R})$, and we would like to estimate the $L_{t, x}^{2}$ norm of the product $e^{i t \Delta} u_{0} e^{i t \Delta} v_{0}$. We assume further that $\varphi$ vanishes in a small neighborhood of the origin.

Remark. The same analysis allows to consider different frequency localizations for $u_{0}$ and $v_{0}$ like $u_{0}=\varphi\left(\sqrt{-\Delta} / N_{1}\right) u_{0}$ and $v_{0}=\psi\left(\sqrt{-\Delta} / N_{2}\right) v_{0}$ with $\varphi, \psi \in C_{0}^{\infty}$ as long as $\varphi$ vanishes in a neighborhood of the origin and $N_{1}$ is sufficiently larger than $N_{2}$. In particular, $\psi$ does not need to vanish near the origin.

To simplify notation, we use $\Delta$ to denote the Laplace-Beltrami operator $\Delta_{g}$ on $M$, and $|\xi|_{g(x)}$ to denote $\sqrt{g(x)^{i j} \xi_{i} \xi_{j}}$.

Proof of Theorem 1.2. The proof is organized as follows. We will first review some important facts about microlocalizing $\varphi(h \sqrt{-\Delta}$ ) and constructing the Schrödinger parametrix (as in [Burq et al. 2004]) that will

\footnotetext{
${ }^{4}$ The full result for $u_{0}, v_{0} \in L^{2}(M)$ can be obtained in the end by a standard limiting argument.
} 
be used to approximate the linear evolutions. The case when $N_{2} \sim N_{1}$, will then follow directly from the semiclassical linear Strichartz estimates already proven in [Burq et al. 2004, Proposition 2.9]. As a result, we will only need to consider the case when $N_{2} \ll N_{1}$. This will ensure that the canonical hypersurfaces associated to the phase functions of the parametrices are transversal as defined in the previous section, a fact which will allow us to apply Theorem 1.1 .

Microlocalizing $\boldsymbol{\varphi}(\boldsymbol{h} \sqrt{-\boldsymbol{\Delta}})$ [Burq et al. 2004; Sogge 1993; Hörmander 1994a; 1994b]. In this section, we will briefly review how spectrally localizing a function $f \in C^{\infty}(M)$ using the spectral multiplier $\varphi(h \sqrt{-\Delta})$ is expressed in local coordinates. Essentially, up to smooth remainder terms, $\varphi(h \sqrt{-\Delta}) f$ is given in local coordinates as a pseudodifferential operator whose symbol $a(x, \xi)$ has a support that reflects the spectral localization dictated by $\varphi$ :

Proposition 3.1. Let $\varphi \in C_{0}^{\infty}(\mathbb{R})$ and $\kappa: U \subset \mathbb{R}^{d} \rightarrow V \subset M$ be a coordinate parametrization of $M$. Also let $\chi_{1}, \chi_{2} \in C_{0}^{\infty}(V)$ be such that $\chi_{2}=1$ near the support of $\chi_{1}$. Then for every $N \in \mathbb{N}$, every $h \in(0,1)$, and every $\sigma \in[0, N]$, there exists $a_{N}(x, \xi)$ supported in $\left\{(x, \xi) \in U \times \mathbb{R}^{d}: \kappa(x) \in \operatorname{supp}\left(\chi_{1}\right),|\xi|_{g(x)} \in \operatorname{supp}(\varphi)\right\}$ such that

$$
\left\|\kappa^{*}\left(\chi_{1} \varphi(h \sqrt{-\Delta}) f\right)-a(x, h D) \kappa^{*}\left(\chi_{2} f\right)\right\|_{H^{\sigma}\left(\mathbb{R}^{d}\right)} \lesssim_{N} h^{N-\sigma}\|f\|_{L^{2}(M)}
$$

for every $f \in C^{\infty}(M)$. In particular, if $\varphi$ is supported away from the origin, then so is the $\xi$ support of $a(x, \xi)$. Here $\kappa^{*}$ is used to denote the pull-back map given by $\kappa^{*} f=f \circ \kappa$.

Proof. See Proposition 2.1 of [Burq et al. 2004] (alternatively, one can use the parametrix expression of the half-wave operator $e^{i t \sqrt{-\Delta}}$ (see [Sogge 1993] for example), along with the expression of $\varphi$ in terms of its Fourier transform).

A consequence of this proposition and a finite partition of unity in $M$, one can split $u_{0}=\varphi(h \sqrt{-\Delta}) u_{0}$ into pieces of the form $\chi_{1} \varphi(h \sqrt{-\Delta}) u_{0}$ and replace each of those pieces (incurring an error that is $\left.O\left(h^{N}\left\|u_{0}\right\|_{L^{2}}\right)\right)$ by $a(x, h D) \kappa^{*}\left(\chi_{2} u_{0}\right)$ which is a compactly supported function in space and is pseudolocalized in frequency in the following sense:

There exists a function $\psi \in C_{0}^{\infty}\left(\mathbb{R}^{d}\right)$ such that for all $h \in(0,1), \sigma>0$, and $N>0$,

$$
\kappa^{*}\left(\chi_{1} \varphi(h \sqrt{-\Delta}) f\right)=\psi(h D) \kappa^{*}\left(\chi_{1} \varphi(h \sqrt{-\Delta}) f\right)+r_{1},
$$

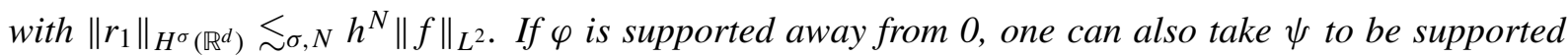
at a positive distance from the origin in $\mathbb{R}^{d}$. This follows easily from Proposition 3.1 and standard pseudodifferential calculus (See [Stein 1993], for example). We will denote $w_{0}(x)=a(x, h D) \kappa^{*}\left(\chi_{2} u_{0}\right)$. In brief, $w_{0}$ is compactly supported in space and can be replaced by $\psi(h D) w_{0}$ at the cost of an error that is $O\left(h^{N}\left\|u_{0}\right\|_{L^{2}(M)}\right)$.

The parametrix [Burq et al. 2004]. With this microlocalization setup, Burq, Gerard, and Tzvetkov constructed an approximate solution in local coordinates to the semiclassical equation

$$
\begin{aligned}
i h \partial_{t} w+h^{2} \Delta_{g} w & =0, \\
w(0) & =\varphi(h \sqrt{-\Delta}) v_{0} .
\end{aligned}
$$


More precisely, using the usual WKB construction (see for example [Hörmander 1994a; 1994b; Burq et al. 2004], or the lecture notes [Evans and Zworski 2003]), they show that there exists $\alpha>0$, such that on the time interval $[-\alpha, \alpha]$

$$
w(s)=\tilde{w}(s)+r_{2}(s),
$$

where $r_{2}(s)$ satisfies $\left\|r_{2}(t)\right\|_{L_{t}^{\infty}\left([-\alpha, \alpha] \times H^{\sigma}(M)\right)} \lesssim h^{N}\left\|w_{0}\right\|_{L^{2}(M)}$ (with $N$ sufficiently large) and $\tilde{w}(t)$ is supported in a compact subset of $V \subset M$ and is given in local coordinates by the oscillatory integral

$$
\tilde{w}(s, x)=\frac{1}{(2 \pi h)^{d}} \int_{\mathbb{R}^{d}} e^{(i / h) \tilde{\phi}(s, x, \xi)} a(s, x, \xi, h) \widehat{w}_{0}\left(\frac{\xi}{h}\right) d \xi .
$$

Here $a(s, x, \xi, h)=\sum_{j=0}^{N} h^{j} a_{j}(s, x, \xi)$, and $a_{j} \in C_{0}^{\infty}\left([-\alpha, \alpha] \times U \times U^{\prime} \Subset \mathbb{R} \times \mathbb{R}^{d} \times \mathbb{R}^{d}\right)$, while $w_{0}$ is the microlocalization of $\varphi(h \sqrt{\Delta}) v_{0}$ described above. Since $w_{0}$ can be replaced by $\psi(h D) w_{0}$ at the cost of an error that is $O\left(h^{N}\left\|w_{0}\right\|_{L^{2}\left(\mathbb{R}^{d}\right)}\right)$ one can assume without loss of generality that $a(s, x, \xi, h)$ has its $\xi$ support at a positive distance from the origin in frequency space if $\varphi$ is supported away from 0 itself.

The phase function $\tilde{\phi}$ appearing in the integral (3-5) satisfies the eikonal equation

$$
\begin{aligned}
\partial_{s} \tilde{\phi}+\sum_{i j} g^{i j} \partial_{i} \tilde{\phi} \partial_{j} \tilde{\phi} & =0, \\
\tilde{\phi}(0, x, \xi) & =x . \xi .
\end{aligned}
$$

Semiclassical linear Strichartz estimates and the case $N_{1} \sim N_{2}$. Using this representation, one can easily use stationary phase (see [Burq et al. 2004] for details) to get the semiclassical dispersion estimate

$$
\left\|e^{i t \Delta} \varphi^{2}(h \sqrt{-\Delta}) v_{0}\right\|_{L^{\infty}(M)} \lesssim_{M} \frac{1}{t^{d / 2}}\left\|v_{0}\right\|_{L^{1}(M)}
$$

for every $t \in[-\alpha h, \alpha h]$ with $0<\alpha \ll 1$. Combining this with the Keel-Tao machinery [1998] one immediately gets the semiclassical Strichartz estimate

$$
\left\|e^{i t \Delta} \varphi(h \sqrt{-\Delta}) u_{0}\right\|_{L_{t}^{q} L_{x}^{r}([-\alpha h, \alpha h] \times M)} \lesssim_{M}\left\|u_{0}\right\|_{L^{2}(M)}
$$

whenever $2 \leqslant q, r \leqslant \infty$ satisfy $2 / q+d / r=d / 2$ and $(q, r, d) \neq(2, \infty, 2)$.

This estimate is enough to prove (1-11) in the case when $h=1 / N_{1} \sim m=1 / N_{2}$. In fact, for $d=2$, one can use the $L_{t, x}^{4}$ Strichartz estimate to get $\left\|e^{i t \Delta} u_{0} e^{i t \Delta} v_{0}\right\|_{L_{t, x}^{2}\left([-\alpha h, \alpha h] \times M^{2}\right)} \leqslant\left\|e^{i t \Delta} \varphi(h \sqrt{-\Delta}) u_{0}\right\|_{L_{t, x}^{4}}\left\|e^{i t \Delta} \varphi(h \sqrt{-\Delta}) v\right\|_{L_{t, x}^{4}} \lesssim\left\|u_{0}\right\|_{L^{2}\left(M^{2}\right)}\left\|v_{0}\right\|_{L^{2}\left(M^{2}\right)}$.

Whereas for $d \geqslant 3$, one can apply Hölder's inequality, the $L_{t}^{\infty} L_{x}^{2}$ bound on $e^{i t \Delta} u_{0}$, Bernstein ${ }^{5}$ and the $L_{t}^{2} L_{x}^{2 d /(d-2)}$ for $e^{i t \Delta} v_{0}$ to get

$$
\left\|e^{i t \Delta} u_{0} e^{i t \Delta} v_{0}\right\|_{L_{t, x}^{2}([0, \alpha h] \times M)} \lesssim N_{2}^{(d-2) / 2}\left\|u_{0}\right\|_{L^{2}(M)}\left\|v_{0}\right\|_{L^{2}(M)}
$$

as desired.

\footnotetext{
${ }^{5}$ One can verify Bernstein's inequality in the setting of compact manifolds by using Proposition 3.2 and the fact that the kernel $K(x, y)$ of $a(x, h D)$ satisfies the bound $\|K(x, y)\|_{L_{x}^{r} L_{y}^{p}\left(\mathbb{R}^{d} \times \mathbb{R}^{d}\right)} \lesssim a h^{-d(1-1 / r-1 / p)}$.
} 
The case $N_{1} \gg N_{2}$. In this section, we will reduce the case $N_{1} \gg N_{2}$ to a verification of the conditions of (1-5). By rescaling time, we have

$$
\begin{aligned}
\left\|e^{i t \Delta} u_{0} e^{i t \Delta} v_{0}\right\|_{L_{t, x}^{2}([-\alpha h, \alpha h] \times M)} & =h^{1 / 2}\left\|e^{i h t \Delta} u_{0} e^{i h t \Delta} v_{0}\right\|_{L_{t, x}^{2}([-\alpha, \alpha] \times M)} \\
& =h^{1 / 2}\left\|e^{i h t \Delta} u_{0} e^{i m(h / m) t \Delta} v_{0}\right\|_{L_{t, x}^{2}([-\alpha, \alpha] \times M)} .
\end{aligned}
$$

As a result it is enough to show

$$
\left\|e^{i h t \Delta} u_{0} e^{i m\left(\frac{h}{m} t\right) \Delta} v_{0}\right\|_{L_{t, x}^{2}([-\alpha, \alpha] \times M)} \lesssim \frac{1}{m^{(d-1) / 2}}\left\|u_{0}\right\|_{L^{2}(M)}\left\|v_{0}\right\|_{L^{2}(M)} .
$$

The advantage of writing the estimate in this way is that we can now use the parametrices for $e^{i t h \Delta_{0}} u_{0}$ and $e^{i t m \Delta} v_{0}$ constructed above to write ${ }^{6}$

$$
e^{i t h \Delta} u_{0}(x)=\tilde{T}_{h} u_{0}(t, x)+R_{h} u_{0}(t, x)
$$

and

$$
e^{i m(h t / m) \Delta} v_{0}(x)=\tilde{S}_{m} v_{0}(t, x)+R_{m} v_{0}(t, x),
$$

where $\tilde{T}_{h}$ and $\tilde{S}_{m}$ are defined according to (3-5) by

$$
\tilde{T}_{h} u_{0}(t, x)=\frac{1}{(2 \pi h)^{d}} \int_{\mathbb{R}^{d}} e^{(i / h) \tilde{\phi}(t, x, \xi)} a_{1}(t, x, \xi, h) \widehat{\tilde{u}_{0}}\left(\frac{\xi}{h}\right) d \xi
$$

and

$$
\tilde{S}_{m} v_{0}(t, x)=\frac{1}{(2 \pi m)^{d}} \int_{\mathbb{R}^{d}} e^{(i / m) \tilde{\phi}\left(h t / m, x, \xi_{2}\right)} a_{2}\left(\frac{h}{m} t, x, \xi_{2}, m\right) \widehat{\tilde{v}}_{0}\left(\frac{\xi_{2}}{m}\right) d \xi_{2},
$$

where $\tilde{u}_{0}$ and $\tilde{v}_{0}$ are the respective microlocalizations of $u_{0}$ and $v_{0}$ in the considered coordinate patch (in particular $\left\|\tilde{u}_{0}\right\|_{L^{2}\left(\mathbb{R}^{d}\right)} \lesssim\left\|u_{0}\right\|_{L^{2}(M)}$ and $\left.\left\|\tilde{v}_{0}\right\|_{L^{2}(M)} \lesssim\left\|v_{0}\right\|_{L^{2}(M)}\right)$. Also we have

$$
\left\|R_{h} u_{0}\right\|_{L_{t}^{\infty} H^{\sigma}([-\alpha, \alpha] \times M)} \lesssim h^{N}\left\|u_{0}\right\|_{L^{2}(M)} \quad \text { and } \quad\left\|R_{m} v_{0}\right\|_{L_{t}^{\infty} H^{\sigma}([-\alpha, \alpha] \times M)} \lesssim m^{N}\left\|v_{0}\right\|_{L^{2}(M)} .
$$

The main contribution comes of course from the product $\tilde{T}_{h} u_{0} \tilde{S} v_{0}$. For example the cross terms $\tilde{T}_{h} u_{0} R_{m} v_{0}$ and $R_{h} u_{0} \tilde{S}_{m} v_{0}$ can be bounded as follows:

$$
\left\|\tilde{T}_{h} u_{0} R_{m} v_{0}\right\|_{L_{t, x}^{2}} \leqslant\left\|\tilde{T}_{h} u_{0}\right\|_{L_{t}^{\infty} L_{x}^{2}}\left\|R_{m} v_{0}\right\|_{L_{t}^{2} L_{x}^{\infty}} \lesssim\left\|u_{0}\right\|_{L^{2}}\left\|v_{0}\right\|_{L^{2}},
$$

where in the last step we used (3-14) and a crude Sobolev embedding to bound $\left\|R_{m} v_{0}\right\|_{L_{t}^{2} L_{x}^{\infty}}$ by $\left\|R_{m}\right\|_{L_{t}^{2} H_{x}^{\sigma}}$ for some $\sigma>d / 2$. The $L_{t}^{\infty} L_{x}^{2}$ bound on $\tilde{T}_{h} u_{0}$ follows from the $L_{t}^{\infty} L_{x}^{2}$ boundedness of $e^{i t h \Delta} u_{0}$. Similarly, one bounds the contributions of $R_{h} u_{0} \tilde{S}_{m} v_{0}$ and $R_{h} u_{0} R_{m} v_{0}$.

To bound the contribution of $\tilde{T}_{h} u_{0} \tilde{S}_{m} v_{0}$, we now apply Theorem 1.1 with $\phi(t, x, \xi)=\tilde{\phi}(t, x, \xi)$ and $\psi\left(t, x, \xi_{2}\right)=\tilde{\phi}\left((h / m) t, x, \xi_{2}\right), f(\xi):=\tilde{u}(\xi / h)$, and $g(\xi)=\tilde{v}_{0}(\xi / m)$, to get

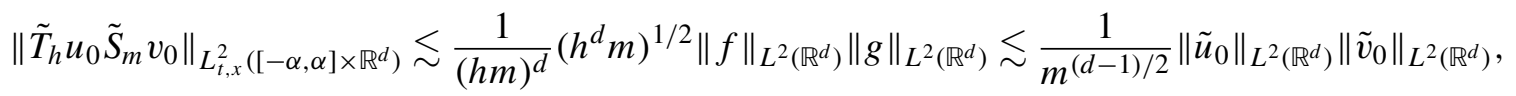

\footnotetext{
${ }^{6}$ Strictly speaking this representation only holds in an open neighborhood of $x_{0} \in M$. Since $M$ is compact, we can cover it by finitely many of such neighborhood, and hence we only need to prove the estimate on each one of them.
} 
which clearly gives (3-11) and hence (1-11). As a result, all we need to do is to verify that the requirements of Theorem 1.1 are satisfied.

Obviously all derivatives of $\phi$ and $\psi$ are uniformly bounded on the compact supports of $a_{1}$ and $a_{2}$ $(h / m \leqslant 1)$. Moreover, since $\tilde{\phi}(0, x, \xi)=x . \xi$, we have that $\left(\partial^{2} \phi / \partial \xi \partial x\right)(0, x, \xi)=$ Id (invertible), the nondegeneracy condition (1-2) is satisfied at $t=0$ and hence for all $t \in[-\alpha, \alpha]$ if $\alpha$ is small enough.

Now we consider the canonical surfaces $S_{\phi}$ and $S_{\psi}$ :

Recall that $S_{\phi}$ and $S_{\psi}$ are the images of the maps

$$
\begin{aligned}
& \xi_{1} \mapsto \nabla_{t, x} \phi\left(t, x, \xi_{1}\right)=\left(\nabla_{x} \tilde{\phi}\left(t, x, \xi_{1}\right), \partial_{t} \tilde{\phi}\left(t, x, \xi_{1}\right)\right), \\
& \xi_{2} \mapsto \nabla_{t, x} \psi\left(t, x, \xi_{2}\right)=\left(\nabla_{x} \tilde{\phi}\left(\frac{h}{m} t, x, \xi_{2}\right), \frac{h}{m} \partial_{t} \tilde{\phi}\left(\frac{h}{m} t, x, \xi_{2}\right)\right),
\end{aligned}
$$

respectively. By the nondegeneracy condition above, $S_{\phi}$ and $S_{\psi}$ are smooth embedded hypersurfaces in $T_{(t, x)}^{*} \mathbb{R}^{d+1}$. We need to show that if $v_{1}\left(\xi_{1}\right)$ is the normal to $S_{\phi}$ at $\nabla_{t, x} \phi\left(t, x, \xi_{1}\right)$ and $v\left(\xi_{2}\right)$ is the normal to $S_{\psi}$ at $\nabla_{t, x} \psi\left(t, x, \xi_{2}\right)$, then there is a $\delta>0$ (uniform in $\xi_{1}$ and $\xi_{2}$ ) such that

$$
\left|\left\langle v_{1}, v_{2}\right\rangle\right| \leqslant 1-\delta
$$

By continuity, we only need to verify (3-15) at $t=0$ for all $x, \xi_{1}, \xi_{2}$. This will imply that the same holds for all $t \in[-\alpha, \alpha]$ if $\alpha$ is small enough. We now fix $\left(0, x_{0}\right) \in \mathbb{R}^{d+1}$ and consider the surfaces $S_{\phi}$ and $S_{\phi}$ in $T_{\left(0, x_{0}\right)}^{*} \mathbb{R}^{d+1}$. From the eikonal equation (3-6), $\tilde{\phi}(0, x, \xi)=x$. $\xi$ and $\partial_{t} \tilde{\phi}(0, x, \xi)=g^{i j}(x) \xi_{i} \xi_{j}$. A straightforward computation gives

$$
\nu_{1}(\xi)=\frac{\left(2 g^{1 j} \xi_{j}, 2 g^{2 j} \xi_{j}, \ldots, 2 g^{d j} \xi_{j},-1\right)}{\sqrt{1+4|\xi|_{g(x)}^{2}}}
$$

and

$$
\nu_{2}(\xi)=\frac{\left(2(h / m) g^{1 j} \xi_{j}, 2(h / m) g^{2 j} \xi_{j}, \ldots, 2(h / m) g^{d j} \xi_{j},-1\right)}{\sqrt{1+4|(h / m) \xi|_{g(x)}^{2}}},
$$

where we recall our notation that $|\xi|_{g(x)}=\sqrt{g(x)^{i j} \xi_{i} \xi_{j}}$. As a result,

$$
\left\langle v_{1}\left(\xi_{1}\right), v_{2}\left(\xi_{2}\right)\right\rangle=\frac{1}{\sqrt{1+4\left|\xi_{1}\right|_{g(x)}^{2}}} \sqrt{1+4\left|(h / m) \xi_{2}\right|_{g(x)}^{2}}+O\left(\frac{h}{m}\right) .
$$

Since $\left|\xi_{1}\right| \gtrsim 1$ and $\left|\xi_{2}\right| \lesssim 1,{ }^{7}$ we get that (3-15) holds true if $h / m$ is small enough.

The proof of (1-12) follows by splitting the time interval $[0, T]$ into pieces of length $N_{1}^{-1}$. That of (1-14) follows by setting $T=1$ in (1-14) when $N_{1} \geqslant 1$ and by using the $L_{t}^{\infty} L_{x}^{2}$ estimates and Sobolev's inequality if $N_{1} \leqslant 1$.

Remark. If $P(D)$ is a differential operator on $M$ of degree $n$, then $P(D) e^{i h t \Delta} u_{0}$ has the expression

$$
P(D) e^{i h t \Delta} u_{0}(x)=h^{-n} \tilde{T}_{h}^{\prime} u_{0}(t, x)+R_{h}^{\prime} u_{0}(t, x),
$$

\footnotetext{
${ }^{7}$ Without loss of generality, we can assume that $\left\|g^{i j}-\delta^{i j}\right\| \leqslant f r a c 1 C$ for some large enough $C$ on the coordinate patch considered. This is enough to have $|\xi|_{g(x)} \sim|\xi|$.
} 
where $\tilde{T}_{h}^{\prime}$ and $R_{h}^{\prime}$ are operators of the same form as $T_{h}$ and $R_{h}$. In particular, $T_{h}^{\prime}$ has an expression as in (3-12) (just with different $a$ ) and $R_{h}^{\prime}$ obeys similar estimates to (3-14) (by choosing $h$ small enough). Similar expressions for $e^{i m t \Delta} v_{0}$ allow us, using the exact same analysis performed above, to get:

Corollary 3.2. Suppose the $u_{0}, v_{0} \in L^{2}(M)$ are spectrally localized around $N_{1}, N_{2} \in 2^{\mathbb{Z}}$ respectively as in Corollary 1.3. Let $P(D)$ and $Q(D)$ be differential operators on $M$ of orders $n$ and $m$ respectively:

$$
\left\|P(D) e^{i t \Delta} u_{0} Q(D) e^{i t \Delta} v_{0}\right\|_{L^{2}([0, T] \times M)} \leqslant N_{1}^{n} N_{2}^{m} \Lambda\left(T, N_{1}, N_{2}\right)\left\|u_{0}\right\|_{L^{2}(M)}\left\|v_{0}\right\|_{L^{2}(M)},
$$

where $\Lambda\left(T, N_{1}, N_{2}\right)$ is given in (1-13).

This variant will be useful in some applications of the bilinear Strichartz estimates proved here (see [Hani 2012] for example).

\section{Further results and remarks}

Bilinear inhomogeneous estimates. Here we will present some inhomogeneous versions of the bilinear estimates proved in the previous section. We will assume that $u(t)$ and $v(t)$ solve the inhomogeneous Schrödinger equation with forcing terms $F$ and $G$ respectively. More precisely,

$$
\begin{aligned}
& i \partial_{t} u+\Delta u=F, \\
& i \partial_{t} v+\Delta v=G .
\end{aligned}
$$

$F$ and $G$ can be assumed to be a priori in $C^{\infty} .^{8}$ The question now is to determine estimates for $\|u v\|_{L_{t, x}^{2}}$ in terms of the initial data $u(0)=u_{0}, v(0)=v_{0}$ and the forcing terms $F$ and $G$.

We will prove two types of inhomogeneous estimates: one corresponding to spectrally localized functions generalizing (1-11) and another is a time $T=1$ estimate generalizing (1-14).

Theorem 4.1. Suppose $u(t)$ and $v(t)$ solve the inhomogeneous Schrödinger equations (4-1) and (4-2) with initial data $u(0)=u_{0}$ and $v(0)=v_{0}$ respectively. Also suppose that $(q, r)$ and $(\tilde{q}, \tilde{r})$ are two Schrödinger admissible exponents.

(i) If $u(t)=\varphi\left(\sqrt{-\Delta} / N_{1}\right) u(t)$ and $v(t)=\varphi\left(\sqrt{-\Delta} / N_{2}\right) v(t)$ for all $t$, then

$$
\|u v\|_{L_{t, x}^{2}\left(\left[0,1 / N_{1}\right] \times M\right)} \lesssim \frac{N_{2}^{(d-1) / 2}}{N_{1}^{1 / 2}}\left(\left\|u_{0}\right\|_{L^{2}(M)}+\|F\|_{L_{t}^{q^{\prime}} L_{x}^{r^{r^{\prime}}}}\right)\left(\left\|v_{0}\right\|_{L^{2}(M)}+\|G\|_{L_{t}^{\tilde{q}^{\prime}} L_{x}^{\tilde{r}^{\prime}}}\right),
$$

where for any $p \in[1, \infty], p^{\prime}$ denotes its conjugate exponent $1 / p+1 / p^{\prime}=1$.

(ii) In general, for any $\delta>0$ we have

$$
\begin{aligned}
& \|u v\|_{L_{t, x}^{2}([0,1] \times M)} \\
& \quad \lesssim\left(\left\|u_{0}\right\|_{H^{\delta}(M)}+\left\|(\sqrt{1-\Delta})^{\delta+1 / q} F\right\|_{L_{t}^{q^{\prime}} L_{x}^{r^{\prime}}}\right)\left(\left\|v_{0}\right\|_{H^{1 / 2-\delta}(M)}+\left\|(\sqrt{1-\Delta})^{1 / 2-\delta+1 / \tilde{q}} G\right\|_{L_{t}^{\tilde{q}^{\prime}} L_{x}^{\tilde{r}^{\prime}}}\right) .
\end{aligned}
$$

For the proof, we will need the Christ-Kiselev lemma [2001], which we state following [Smith and Sogge 2000]:

\footnotetext{
${ }^{8}$ This assumption can be removed a posteriori using standard density arguments.
} 
Lemma 4.2. Let $X$ and $Y$ be Banach spaces and $K(t, x)$ a continuous function taking values in $B(X, Y)$, the space of bounded linear mappings from $X$ to $Y$. Suppose that $-\infty \leqslant a<b \leqslant \infty$ and let

$$
T f(t)=\int_{a}^{b} K(t, s) f(s) d s .
$$

Suppose that

$$
\|T f\|_{L^{q}([a, b] ; Y)} \leqslant C\|f\|_{L^{p}([a, b] ; X)},
$$

and define the lower triangular operator

$$
W f(t)=\int_{a}^{t} K(t, s) f(s) d s .
$$

Then, if $1 \leqslant p<q \leqslant \infty$,

$$
\|W f\|_{L^{q}([a, b] ; Y)} \lesssim C\|f\|_{L^{p}([a, b] ; X)} .
$$

Proof of Theorem 4.1. We start by proving the spectrally localized version in (4-3). The integral equations satisfied by $u(t)$ and $v(t)$ are given by Duhamel's formula:

$$
u(t)=e^{i t \Delta} u_{0}-i \int_{0}^{t} e^{i(t-s) \Delta} F(s) d s \quad \text { and } \quad v(t)=e^{i t \Delta} v_{0}-i \int_{0}^{t} e^{i(t-s) \Delta} G(s) d s .
$$

As a result,

$$
\begin{aligned}
u(t) v(t)=e^{i t \Delta} u_{0} e^{i t \Delta} v_{0} & -i e^{i t \Delta} u_{0} \int_{0}^{t} e^{i(t-s) \Delta} G(s) d s \\
& -i e^{i t \Delta} v_{0} \int_{0}^{t} e^{i(t-s) \Delta} F(s) d s-\int_{0}^{t} e^{i(t-s) \Delta} F(s) d s \int_{0}^{t} e^{i(t-r) \Delta} G(r) d r .
\end{aligned}
$$

Recall that $u_{0}, u(t), F(t)$ are all spectrally localized at dyadic scale $N_{1}$ and $v_{0}, v(t), G(t)$ localized at scale $N_{2}$. The estimate for the first term on the right in (4-5) is the bilinear Strichartz estimate proved in the previous section. We turn to the second term. Applying the Christ-Kiselev lemma (with $Y=L_{t}^{\tilde{q}^{\prime}} L_{x}^{\tilde{r}^{\prime}}$, $X=L_{t, x}^{2}\left(\left[0,1 / N_{1}\right] \times M\right)$, and $\left.C \sim N_{2}^{(d-1) / 2} / N_{1}^{1 / 2}\left\|u_{0}\right\|_{L^{2}(M)}\right)$, it is enough to show

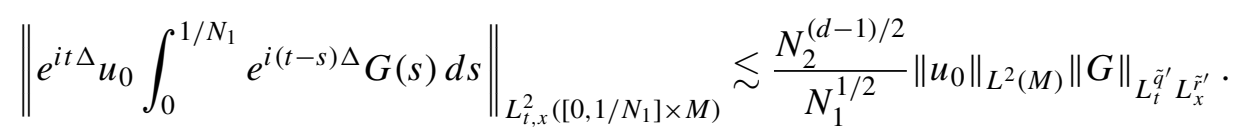

But this follows from the bilinear estimate (1-11) and

$$
\left\|\int_{0}^{1 / N_{1}} e^{-i s \Delta} \varphi\left(\frac{\sqrt{-\Delta}}{N_{1}}\right) G(s) d s\right\|_{L_{x}^{2}(M)} \lesssim\|G\|_{L_{t}^{\tilde{q}^{\prime}} L_{x}^{\tilde{r}^{\prime}}},
$$

which is the dual estimate to (1-9). 
The third term on the right in (4-5) is estimated similarly. For the fourth term, we first apply the Christ-Kiselev lemma to reduce the estimate to

$$
\begin{aligned}
& \left\|\int_{0}^{1 / N_{1}} e^{i(t-s) \Delta} F(s) d s \int_{0}^{t} e^{i(t-r) \Delta} G(r) d r\right\|_{L_{t, x}^{2}\left(\left[0,1 / N_{1}\right] \times M\right)} \\
& =\left\|e^{i t \Delta}\left(\int_{0}^{1 / N_{1}} e^{-i s \Delta} F(s) d s\right) \int_{0}^{t} e^{i(t-r) \Delta} G(r) d r\right\|_{L_{t, x}^{2}} \\
& \lesssim \frac{N_{2}^{(d-1) / 2}}{N_{1}}\left\|\int_{0}^{N_{1}^{-1}} e^{-i s \Delta} F(s) d s\right\|_{L^{2}(M)}\|G\|_{L_{t}^{\tilde{q}^{\prime}} L_{x}^{\tilde{r}^{\prime}}} \lesssim\|F\|_{L_{t}^{q^{\prime}} L_{x}^{r^{\prime}}}\|G\|_{L_{t}^{\tilde{q}^{\prime}} L_{x}^{\tilde{r}^{\prime}}},
\end{aligned}
$$

where in the first inequality we apply the same analysis as that used to estimate the second and third term on the right in (4-5) (or apply Christ-Kiselev lemma again) while in the second we use the dual homogeneous Strichartz estimate. This finishes the proof of (4-3).

We now turn to the time 1 estimate (4-4). We start by mentioning that the first term on the right in (4-5) satisfies the needed estimate

$$
\left\|e^{i t \Delta} u_{0} e^{i t \Delta} v_{0}\right\|_{L^{2}([0,1] \times M)} \lesssim\left\|u_{0}\right\|_{H^{\delta}}\left\|v_{0}\right\|_{H^{1 / 2-\delta}}
$$

This follows directly by splitting into Littlewood-Paley pieces $u=\sum_{\substack{N_{1} \geqslant 1 \\ \text { (dyadic) }}} u_{N_{1}}$ and $v=\sum_{\substack{N_{2} \geqslant 1 \\ \text { (dyadic) }}} v_{N_{2}}$ and
estimating by $\left\|e^{i t \Delta} u_{0} e^{i t \Delta} v_{0}\right\|_{L_{t, x}^{2}([0,1] \times M)}$

$$
\begin{aligned}
& \lesssim \sum_{N_{1} \leqslant N_{2}}\left\|e^{i t \Delta} u_{N_{1}} e^{i t \Delta} v_{N_{2}}\right\|_{L_{t, x}^{2}}+\sum_{N_{1}>N_{2}}\left\|e^{i t \Delta} u_{N_{1}} e^{i t \Delta} v_{N_{2}}\right\|_{L_{t, x}^{2}} \\
& \lesssim \sum_{N_{1} \leqslant N_{2}} N_{1}^{(d-1) / 2}\left\|u_{N_{1}}\right\|_{L^{2}}\left\|v_{N_{2}}\right\|_{L^{2}}+\sum_{N_{2}<N_{1}} N_{2}^{(d-1) / 2}\left\|u_{N_{1}}\right\|_{L^{2}}\left\|v_{N_{2}}\right\|_{L^{2}} \\
& \lesssim \sum_{N_{1} \leqslant N_{2}} \frac{N_{1}^{(d-1) / 2-\delta}}{N_{2}^{(d-1) / 2-\delta}}\left\|u_{N_{1}}\right\|_{H^{\delta}}\left\|v_{N_{2}}\right\|_{H^{(d-1) / 2-\delta}}+\sum_{N_{2}<N_{1}} \frac{N_{2}^{\delta}}{N_{1}^{\delta}}\left\|u_{N_{1}}\right\|_{H^{\delta}}\left\|u_{N_{2}}\right\|_{H^{(d-1) / 2-\delta}} \\
& \lesssim\|u\|_{H^{\delta}}\|v\|_{H^{(d-1) / 2-\delta}},
\end{aligned}
$$

where we have used Schur's test to sum in the last step. The rest of the proof of (4-4) follows as that of (4-3) above except that here we use the estimate dual to (1-8) given by

$$
\left\|\int_{0}^{1} e^{i(t-s) \Delta} F(s) d s\right\|_{L^{2}(M)} \lesssim\left\|(\sqrt{1-\Delta})^{1 / q} F\right\|_{L_{t}^{q^{\prime}} L_{x}^{r^{\prime}([0,1] \times M)}} .
$$

Bilinear estimates of mixed type. Here we present an instance of a mixed-type bilinear estimate of Schrödinger-wave type that can be proved using Theorem 1.1. Constant coefficient versions of such estimates are often useful when studying coupled Schrödinger-wave systems such as the Zakharov system (see [Bejenaru et al. 2009] for instance). Theorem 4.3 below serves as an example of a variable coefficient 
Schrödinger-wave bilinear estimates and has potential applications in studying Zakharov systems (or other Schrödinger-wave systems) on manifolds.

Theorem 4.3. Suppose $u_{0}, v_{0} \in L^{2}\left(M^{d}\right)$ are spectrally localized at dyadic scales $N_{1}$ and $N_{2}$ as above with $1 \ll N_{1}$. Then the estimate

$$
\left\|e^{i t \Delta} u_{0} e^{ \pm i t|\nabla|} v_{0}\right\|_{L_{t, x}^{2}\left(\left[-1 / N_{1}, 1 / N_{1}\right] \times M\right)} \lesssim M \frac{\min \left(N_{1}, N_{2}\right)^{(d-1) / 2}}{N_{1}^{1 / 2}}\left\|u_{0}\right\|_{L^{2}(M)}\|v\|_{L^{2}(M)}
$$

holds. Of course, an estimate over the time interval $[0, T]$ follows as well by splitting into pieces of length $1 / N_{1}$.

Proof. We present the proof in the case of the forward half wave operator, the proof for the backwards operator being similar. As before, we use the parametrix for $e^{i t|\nabla|} v_{0}$ which is given, up to a smoothing remainder $R_{m} v_{0}$, by the oscillatory integral

$$
S_{m}^{W} v_{0}=\frac{1}{(2 \pi m)^{d}} \int_{\mathbb{R}^{d}} e^{(i / m) \psi\left(t, x, \xi_{2}\right)} a\left(t, x, \xi_{2}\right) \widetilde{\tilde{v}}_{0}\left(\frac{\xi_{2}}{m}\right) d \xi_{2}
$$

where $\psi$ is a nondegenerate phase function (in particular $\left.\operatorname{det}\left(\left(\partial^{2} / \partial \xi \partial x\right) \tilde{\psi}\right) \neq 0\right)$ and homogeneous in $\xi_{2}$ of degree 1 and $\tilde{v}_{0}$ is a microlocalization of $v_{0}$ as explained in Section 3 (cf. [Hörmander 1994b, Chapter XXIX]). As before, we used the convention that $h=1 / N_{1}$ and $m=1 / N_{2}$. As a result, we have

$$
\left\|e^{i t \Delta} u_{0} e^{i t|\nabla|} v_{0}\right\|_{L_{t, x}^{2}\left(\left[-\alpha / N_{1}, \alpha / N_{1}\right] \times M\right)}=h^{1 / 2}\left\|e^{i h t \Delta} u_{0} e^{i h t|\nabla|} v_{0}\right\|_{L_{t, x}^{2}([-\alpha, \alpha] \times M)} .
$$

Ignoring the smooth remainder terms $R_{h}$ and $R_{m}$ (as they are inconsequential as in Section 3) we get that (4-6) follows from the estimate

$$
\begin{aligned}
&\left\|\tilde{T}_{h} u_{0}(t, x) \tilde{S}_{m}^{W} v_{0}(h t, x)\right\|_{L_{t, x}^{2}\left([-\alpha, \alpha] \times \mathbb{R}^{d}\right)} \lesssim \frac{1}{(h m)^{d / 2}} \min (m, h)^{d / 2} \max (m, h)^{1 / 2}\left\|\tilde{u}_{0}\right\|_{L^{2}\left(\mathbb{R}^{d}\right)}\left\|\tilde{v}_{0}\right\|_{L^{2}\left(\mathbb{R}^{d}\right)} \\
&=C \max (m, h)^{-(d-1) / 2}\left\|\tilde{u}_{0}\right\|_{L^{2}\left(\mathbb{R}^{d}\right)}\left\|\tilde{v}_{0}\right\|_{L^{2}\left(\mathbb{R}^{d}\right)} .
\end{aligned}
$$

This inequality follows by applying Equation (1-5) with the nondegenerate phase functions $\phi\left(t, x, \xi_{1}\right)=$ $\tilde{\phi}\left(t, x, \xi_{1}\right)$ and $\psi\left(t, x, \xi_{2}\right)=\tilde{\psi}\left(h t, x, \xi_{2}\right)$. The transversality condition is directly verified as follows. The normal vectors to the two surfaces

$$
\begin{aligned}
S_{\phi}: \xi_{1} \mapsto \nabla_{t, x} \phi\left(t, x, \xi_{1}\right) & =\left(\nabla_{x} \tilde{\phi}\left(t, x, \xi_{1}\right), \partial_{t} \tilde{\phi}\left(t, x, \xi_{1}\right)\right), \\
S_{\psi}: \xi_{2} \mapsto \nabla_{t, x} \psi\left(t, x, \xi_{2}\right) & =\left(\nabla_{x} \tilde{\psi}\left(h t, x, \xi_{2}\right), h \partial_{t} \tilde{\psi}\left(h t, x, \xi_{2}\right)\right)
\end{aligned}
$$

can be written as $\nu_{1}=\left(\eta_{1}, \tau_{1}\right)$ and $\nu_{2}=\left(\eta_{2}, \tau_{2}\right)$ with $\eta_{1}, \eta_{2} \in \mathbb{R}^{n}$ and $\tau_{1}, \tau_{2} \in \mathbb{R}$. The fact that $\left\langle v_{2},\left(\partial^{2} / \partial \xi \partial(x, t)\right) \psi\right\rangle=\overrightarrow{0}$ implies that $\left\langle\eta_{2},\left(\partial^{2} / \partial \xi \partial x\right) \tilde{\psi}\left(h t, x, \xi_{2}\right)\right\rangle+h \tau_{2} \partial_{t} \partial_{\xi} \tilde{\psi}\left(h t, x, \xi_{2}\right)=\overrightarrow{0}$, which implies that

$$
\eta_{2}=-h \tau_{2}\left\langle\partial_{t} \partial_{\xi} \tilde{\psi},\left[\frac{\partial^{2}}{\partial \xi \partial x} \tilde{\psi}\right]^{-1}\right\rangle=O(h)
$$

This gives that

$$
\left\langle v_{1}, v_{2}\right\rangle \leqslant\left|\tau_{1} \tau_{2}\right|+O(h) \leqslant\left|\tau_{1}\right|+O(h) .
$$


As a result, the transversality condition (1-6) holds if $h \ll 1$ (i.e., $N_{1} \gg 1$ ) and $\left|\tau_{1}\right|<1$, which is the case since $\tau_{1}=-1 / \sqrt{1+4|\xi|_{g(x)}^{2}}$ and $\left|\xi_{1}\right| \gtrsim 1$ (see end of the proof of Theorem 1.2).

Applications in PDE. The bilinear estimate (1-14) directly implies local well-posedness for 2-dimensional cubic NLS

$$
\begin{aligned}
i \partial_{t} u+\Delta u & =|u|^{2} u, \\
u(t=0) & =u_{0} \in H^{s}\left(M^{2}\right)
\end{aligned}
$$

in $X^{s, b} \subset C_{t} H_{x}^{s}$ spaces for all $s>1 / 2$ and some $b>\frac{1}{2}$. It should be noted that local well-posedness of (4-7) in $C_{t} H^{s}$ for $s>\frac{1}{2}$ has already been proven in [Burq et al. 2004] using linear Strichartz estimates. Here $X^{s, b}$ is the closure of $C_{0}^{\infty}(\mathbb{R} \times M)$ in the norm

$$
\|u\|_{X^{s, b}}=\left(\int_{\mathbb{R}} \sum_{\nu}\langle\tau+v\rangle^{2 b}\langle v\rangle^{s}\left\|\widehat{\pi_{v} u}(\tau)\right\|_{L^{2}(M)}^{2} d \tau\right)^{1 / 2},
$$

where the sum runs over the distinct eigenvalues of the Laplacian and $\pi_{v}$ is the projection onto the eigenspace corresponding to the eigenvalue $v$. It is worth remarking that (1-11) translates into the following estimate for functions $u, v \in C_{0}^{\infty}(\mathbb{R} \times M)$ satisfying $u(t)=\mathbf{1}_{\left[N_{1}, 2 N_{1}\right)}(\sqrt{-\Delta}) u(t)$ and $v(t)=$ $\mathbf{1}_{\left[N_{2}, 2 N_{2}\right)}(\sqrt{-\Delta}) v(t)$ :

$$
\|u v\|_{L^{2}(\mathbb{R} \times M)} \lesssim \min \left(N_{2}, N_{1}\right)^{1 / 2}\|u\|_{X^{0, b}}\|v\|_{X^{0, b}}
$$

for any $b>\frac{1}{2}$ (cf. [Burq et al. 2005a; Hani 2012]). Using this and a standard dyadic decomposition one can prove the crucial cubic estimate that yields local well-posedness via Picard iteration (see [Burq et al. 2005a] for example).

One interesting application of Theorem 1.2 is that of proving global well-posedness of (4-7) for $s<1$. As mentioned in the introduction, the bilinear Strichartz estimate (1-12) on the time interval [0,T] translates into a bilinear Strichartz estimate on the rescaled manifold $\lambda M$ over the time interval $[0,1]$. Here $\lambda M$ can either be viewed as the Riemmannian manifold $\left(M,\left(1 / \lambda^{2}\right) g\right)$ or by embedding $M$ into some ambient space $R^{N}$ and then applying a dilation by $\lambda$ to get $\lambda M$. The relevant result was cited in the introduction in Corollary 1.3: if $u_{0}, v_{0} \in L^{2}(\lambda M)$ are spectrally localized around $N_{1}$ and $N_{2}$ respectively, with $N_{2} \leqslant N_{1}$. Then

$$
\begin{aligned}
&\left\|e^{i t \Delta_{\lambda}} u_{0} e^{i t \Delta_{\lambda}} v_{0}\right\|_{L^{2}([0,1] \times \lambda M)} \lesssim \Lambda\left(\lambda^{-2}, \lambda N_{1}, \lambda N_{2}\right)\left\|u_{0}\right\|_{L^{2}(\lambda M)}\left\|v_{0}\right\|_{L^{2}(\lambda M)} \\
& \lesssim \begin{cases}\left(N_{2} / N_{1}\right)^{1 / 2}\left\|u_{0}\right\|_{L^{2}(\lambda M)}\left\|v_{0}\right\|_{L^{2}(\lambda M)} & \text { if } \lambda \gg N_{1}, \\
\left(N_{2} / \lambda\right)^{1 / 2}\left\|u_{0}\right\|_{L^{2}(\lambda M)}\left\|v_{0}\right\|_{L^{2}(\lambda M)} & \text { if } \lambda \lesssim N_{1} .\end{cases}
\end{aligned}
$$

This estimate turns out to be crucial in [Hani 2012] where it is proved that (4-7) is globally well-posed for all $s>\frac{2}{3}$. This generalizes, without any loss in regularity, a similar result from [Bourgain 2004] (see also [De Silva et al. 2007]), where global well-posedness for $s>\frac{2}{3}$ is proved for the torus $\mathbb{T}^{2}$. Global well-posedness for $s \geqslant 1$ follows using conservation of energy and standard arguments. To go below the energy regularity $s=1$, the I-method of Colliander, Keel, Staffilani, Takaoka, and Tao should be used 
and most of the analysis is done on $\lambda M$ rather than $M$. As a result, the factor of $1 / \lambda^{1 / 2}$ on the right side of (1-16) in the range $\lambda \lesssim N_{1}$ becomes crucial to get the full regularity range of $s>\frac{2}{3}$ (see [Hani 2012]).

\section{Acknowledgements}

The author is deeply grateful to his advisor, Prof. Terence Tao, for his invaluable support, encouragement, and guidance. He also wishes to extend his immense gratitude to the referee for his careful review of the manuscript and his helpful comments and suggestions that considerably improved and clarified the exposition.

\section{References}

[Bejenaru et al. 2009] I. Bejenaru, S. Herr, J. Holmer, and D. Tataru, "On the 2D Zakharov system with $L^{2}$-Schrödinger data", Nonlinearity 22:5 (2009), 1063-1089. MR 2010f:35383 Zbl 1173.35651

[Bourgain 1993] J. Bourgain, "Fourier transform restriction phenomena for certain lattice subsets and applications to nonlinear evolution equations, I: Schrödinger equations", Geom. Funct. Anal. 3:2 (1993), 107-156. MR 95d:35160a Zbl 0787.35097

[Bourgain 1998] J. Bourgain, "Refinements of Strichartz' inequality and applications to 2D-NLS with critical nonlinearity", Internat. Math. Res. Notices 1998:5 (1998), 253-283. MR 99f:35184 Zbl 0917.35126

[Bourgain 1999] J. Bourgain, Global solutions of nonlinear Schrödinger equations, American Mathematical Society Colloquium Publications 46, American Mathematical Society, Providence, RI, 1999. MR 2000h:35147 Zbl 0933.35178

[Bourgain 2004] J. Bourgain, "A remark on normal forms and the "I-method" for periodic NLS", J. Anal. Math. 94 (2004), 125-157. MR 2006b:37155 Zbl 1084.35085

[Burq et al. 2004] N. Burq, P. Gérard, and N. Tzvetkov, "Strichartz inequalities and the nonlinear Schrödinger equation on compact manifolds", Amer. J. Math. 126:3 (2004), 569-605. MR 2005h:58036 Zbl 1067.58027

[Burq et al. 2005a] N. Burq, P. Gérard, and N. Tzvetkov, "Bilinear eigenfunction estimates and the nonlinear Schrödinger equation on surfaces", Invent. Math. 159:1 (2005), 187-223. MR 2005m:35275 Zbl 1092.35099

[Burq et al. 2005b] N. Burq, P. Gérard, and N. Tzvetkov, "Multilinear eigenfunction estimates and global existence for the three dimensional nonlinear Schrödinger equations”, Ann. Sci. École Norm. Sup. (4) 38:2 (2005), 255-301. MR 2006m:35337 Zbl 1116.35109

[Christ and Kiselev 2001] M. Christ and A. Kiselev, "Maximal functions associated to filtrations", J. Funct. Anal. 179:2 (2001), 409-425. MR 2001i:47054 Zbl 0974.47025

[De Silva et al. 2007] D. De Silva, N. Pavlović, G. Staffilani, and N. Tzirakis, "Global well-posedness for a periodic nonlinear Schrödinger equation in 1D and 2D”, Discrete Contin. Dyn. Syst. 19:1 (2007), 37-65. MR 2008g:35191 Zbl 05236234

[Evans and Zworski 2003] L. C. E. Evans and M. Zworski, "Lectures on semiclassical analysis", University of California, Berkeley, CA, 2003, Available at http://math.berkeley.edu/ evans/semiclassical.pdf.

[Hani 2012] Z. Hani, "Global well-posedness of the $2 D$-cubic nonlinear Schrödinger equation on compact manifolds without boundary”, Comm. Partial Differential Equations 37:7 (2012), 1186-1236. arXiv 1008.2826

[Hörmander 1973] L. Hörmander, "Oscillatory integrals and multipliers on F L p", Ark. Mat. 11 (1973), 1-11. MR 49 \#5674 Zbl 0254.42010

[Hörmander 1994a] L. Hörmander, The analysis of linear partial differential operators, III: Pseudo-differential operators, Grundlehren der Mathematischen Wissenschaften 274, Springer, Berlin, 1994. MR 95h:35255 Zbl 0601.35001

[Hörmander 1994b] L. Hörmander, The analysis of linear partial differential operators, IV: Fourier integral operators, Grundlehren der Mathematischen Wissenschaften 275, Springer, Berlin, 1994. MR 98f:35002 Zbl 0612.35001

[Jiang 2011] J.-C. Jiang, "Bilinear Strichartz estimates for Schrödinger operators in two-dimensional compact manifolds with boundary and cubic NLS", Differential Integral Equations 24:1-2 (2011), 83-108. MR 2012a:35332 Zbl 05944786 
[Keel and Tao 1998] M. Keel and T. Tao, "Endpoint Strichartz estimates", Amer. J. Math. 120:5 (1998), 955-980. MR 2000d: 35018 Zbl 0922.35028

[Lee 2006] S. Lee, "Linear and bilinear estimates for oscillatory integral operators related to restriction to hypersurfaces", $J$. Funct. Anal. 241:1 (2006), 56-98. MR 2007g:42024 Zbl 1121.35151

[Smith and Sogge 2000] H. F. Smith and C. D. Sogge, "Global Strichartz estimates for nontrapping perturbations of the Laplacian”, Comm. Partial Differential Equations 25:11-12 (2000), 2171-2183. MR 2001j:35180 Zbl 0972.35014

[Sogge 1993] C. D. Sogge, Fourier integrals in classical analysis, Cambridge Tracts in Mathematics 105, Cambridge University Press, Cambridge, 1993. MR 94c:35178 Zbl 0783.35001

[Staffilani and Tataru 2002] G. Staffilani and D. Tataru, "Strichartz estimates for a Schrödinger operator with nonsmooth coefficients", Comm. Partial Differential Equations 27:7-8 (2002), 1337-1372. MR 2003f:35248 Zbl 1010.35015

[Stein 1993] E. M. Stein, Harmonic analysis: real-variable methods, orthogonality, and oscillatory integrals, Princeton Mathematical Series 43, Princeton University Press, Princeton, NJ, 1993. MR 95c:42002 Zbl 0821.42001

[Tao 2001] T. Tao, "Endpoint bilinear restriction theorems for the cone, and some sharp null form estimates", Math. Z. 238:2 (2001), 215-268. MR 2003a:42010 Zbl 0992.42004

[Tao 2003] T. Tao, “A sharp bilinear restriction estimate for paraboloids", Geom. Funct. Anal. 13:6 (2003), 1359-1384. MR 2004m:47111 Zbl 1068.42011

[Tao 2004] T. Tao, "Some recent progress on the restriction conjecture", pp. 217-243 in Fourier analysis and convexity, edited by L. Brandolini et al., Birkhäuser, Boston, 2004. MR 2005i:42015 Zbl 1083.42008 arXiv math/0311181

[Tao 2006] T. Tao, Nonlinear dispersive equations: local and global analysis, CBMS Regional Conference Series in Mathematics 106, American Mathematical Society, Providence, RI, 2006. MR 2008i:35211 Zbl 1106.35001

[Wolff 2001] T. Wolff, "A sharp bilinear cone restriction estimate”, Ann. of Math. (2) 153:3 (2001), 661-698. MR 2002j:42019 Zbl 1125.42302

Received 16 Aug 2010. Revised 13 Jan 2011. Accepted 13 Feb 2011.

ZAHER HANI: zhani@math.ucla.edu

Mathematics Department, University of California, Los Angeles, 520 Portola Plaza, Math Sciences Building,

Los Angeles, CA 90095, United States

http://www.math.ucla.edu/ zhani 


\title{
Analysis \& PDE
}

\author{
msp.berkeley.edu/apde
}

EDITORS

EDITOR-IN-CHIEF

Maciej Zworski

University of California

Berkeley, USA

BOARD OF EDITORS

\begin{tabular}{|c|c|c|c|}
\hline Michael Aizenman & $\begin{array}{l}\text { Princeton University, USA } \\
\text { aizenman@math.princeton.edu }\end{array}$ & Nicolas Burq & $\begin{array}{l}\text { Université Paris-Sud 11, France } \\
\text { nicolas.burq@math.u-psud.fr }\end{array}$ \\
\hline Luis A. Caffarelli & $\begin{array}{l}\text { University of Texas, USA } \\
\text { caffarel@math.utexas.edu }\end{array}$ & un-Yung Alice Chang & $\begin{array}{l}\text { Princeton University, USA } \\
\text { chang@math.princeton.edu }\end{array}$ \\
\hline Michael Christ & $\begin{array}{l}\text { University of California, Berkeley, USA } \\
\text { mchrist@math.berkeley.edu }\end{array}$ & Charles Fefferman & $\begin{array}{l}\text { Princeton University, USA } \\
\text { cf@math.princeton.edu }\end{array}$ \\
\hline Ursula Hamenstaedt & $\begin{array}{l}\text { Universität Bonn, Germany } \\
\text { ursula@math.uni-bonn.de }\end{array}$ & Nigel Higson & $\begin{array}{l}\text { Pennsylvania State Univesity, USA } \\
\text { higson@ math.psu.edu }\end{array}$ \\
\hline Vaughan Jones & $\begin{array}{l}\text { University of California, Berkeley, USA } \\
\text { vfr@math.berkeley.edu }\end{array}$ & Herbert Koch & $\begin{array}{l}\text { Universität Bonn, Germany } \\
\text { koch@math.uni-bonn.de }\end{array}$ \\
\hline Izabella Laba & $\begin{array}{l}\text { University of British Columbia, Canada } \\
\text { ilaba@math.ubc.ca }\end{array}$ & Gilles Lebeau & $\begin{array}{l}\text { Université de Nice Sophia Antipolis, France } \\
\text { lebeau@unice.fr }\end{array}$ \\
\hline László Lempert & $\begin{array}{l}\text { Purdue University, USA } \\
\text { lempert@math.purdue.edu }\end{array}$ & Richard B. Melrose & $\begin{array}{l}\text { Massachussets Institute of Technology, USA } \\
\text { rbm@math.mit.edu }\end{array}$ \\
\hline Frank Merle & $\begin{array}{l}\text { Université de Cergy-Pontoise, France } \\
\text { Frank.Merle@u-cergy.fr }\end{array}$ & William Minicozzi II & $\begin{array}{l}\text { Johns Hopkins University, USA } \\
\text { minicozz@ math.jhu.edu }\end{array}$ \\
\hline Werner Müller & $\begin{array}{l}\text { Universität Bonn, Germany } \\
\text { mueller@math.uni-bonn.de }\end{array}$ & Yuval Peres & $\begin{array}{l}\text { University of California, Berkeley, USA } \\
\text { peres@stat.berkeley.edu }\end{array}$ \\
\hline Gilles Pisier & $\begin{array}{l}\text { Texas A\&M University, and Paris } 6 \\
\text { pisier@math.tamu.edu }\end{array}$ & Tristan Rivière & $\begin{array}{l}\text { ETH, Switzerland } \\
\text { riviere@math.ethz.ch }\end{array}$ \\
\hline Igor Rodnianski & $\begin{array}{l}\text { Princeton University, USA } \\
\text { irod@math.princeton.edu }\end{array}$ & Wilhelm Schlag & $\begin{array}{l}\text { University of Chicago, USA } \\
\text { schlag@math.uchicago.edu }\end{array}$ \\
\hline Sylvia Serfaty & $\begin{array}{l}\text { New York University, USA } \\
\text { serfaty@ cims.nyu.edu }\end{array}$ & Yum-Tong Siu & $\begin{array}{l}\text { Harvard University, USA } \\
\text { siu@math.harvard.edu }\end{array}$ \\
\hline Terence Tao & $\begin{array}{l}\text { University of California, Los Angeles, USA } \\
\text { tao@math.ucla.edu }\end{array}$ & A Michael E. Taylor & $\begin{array}{l}\text { Univ. of North Carolina, Chapel Hill, USA } \\
\text { met@math.unc.edu }\end{array}$ \\
\hline Gunther Uhlmann & $\begin{array}{l}\text { University of Washington, USA } \\
\text { gunther@math.washington.edu }\end{array}$ & András Vasy & $\begin{array}{l}\text { Stanford University, USA } \\
\text { andras@math.stanford.edu }\end{array}$ \\
\hline Virgil Voiculescu & $\begin{array}{l}\text { University of California, Berkeley, USA } \\
\text { dvv@math.berkeley.edu }\end{array}$ & Steven Zelditch & $\begin{array}{l}\text { Northwestern University, USA } \\
\text { zelditch@math.northwestern.edu }\end{array}$ \\
\hline
\end{tabular}

\section{PRODUCTION}

contact@msp.org

Silvio Levy, Scientific Editor

Sheila Newbery, Senior Production Editor

See inside back cover or msp.berkeley.edu/apde for submission instructions.

The subscription price for 2012 is US \$140/year for the electronic version, and \$240/year for print and electronic. Subscriptions, requests for back issues from the last three years and changes of subscribers address should be sent to Mathematical Sciences Publishers, Department of Mathematics, University of California, Berkeley, CA 94720-3840, USA.

Analysis \& PDE, at Mathematical Sciences Publishers, Department of Mathematics, University of California, Berkeley, CA 94720-3840 is published continuously online. Periodical rate postage paid at Berkeley, CA 94704, and additional mailing offices.

APDE peer review and production are managed by EditFLOW ${ }^{\mathrm{TM}}$ from Mathematical Sciences Publishers.

PUBLISHED BY

mathematical sciences publishers

http://msp.org/

A NON-PROFIT CORPORATION

Typeset in IATEX

Copyright $(2012$ by Mathematical Sciences Publishers 


\section{ANALYSIS \& PDE}

\section{Volume $5 \quad$ No. $2 \quad 2012$}

The geodesic X-ray transform with fold caustics

Plamen STEFANOV and GUNTHER UHLMANN

Existence of extremals for a Fourier restriction inequality

Michael Christ and Shuanglin ShaO

Dispersion and controllability for the Schrödinger equation on negatively curved manifolds

NALINI ANANTHARAMAN and GABRIEL RIVIÈRE

A bilinear oscillatory integral estimate and bilinear refinements to Strichartz estimates on closed manifolds

\section{ZAHER HANI}

The Cauchy problem for the Benjamin-Ono equation in $L^{2}$ revisited

LUC MOLINET and DidIER PILOD

On triangles determined by subsets of the Euclidean plane, the associated bilinear operators 